\title{
The Hydrodynamic Coefficient Analysis and Motion Control of the Lingyun Moveable Lander
}

\author{
Guoxin $\operatorname{Li} \mathbb{D}^{1,2}$ and Shaowei Zhang $\mathbb{D}^{1}$ \\ ${ }^{1}$ Institute of Deep-Sea Science and Engineering, Chinese Academy of Sciences, Sanya 572000, China \\ ${ }^{2}$ College of Materials Science and Opto-Electronic Technology, University of Chinese Academy of Sciences, Beijing 100049, China
}

Correspondence should be addressed to Shaowei Zhang; zhangshaowei@idsse.ac.cn

Received 6 July 2021; Revised 27 August 2021; Accepted 8 September 2021; Published 7 October 2021

Academic Editor: Xudong Zhang

Copyright ( ) 2021 Guoxin Li and Shaowei Zhang. This is an open access article distributed under the Creative Commons Attribution License, which permits unrestricted use, distribution, and reproduction in any medium, provided the original work is properly cited.

\begin{abstract}
A moveable lander has the advantages of low cost and strong controllability and is gradually becoming an effective autonomous ocean observation platform. In this study, the hydrodynamic property of the Lingyun moveable lander, which has completed experiments in the Mariana Trench in 2020, is analyzed with the semiempirical method and computational fluid dynamic (CFD) method. We calculate the inertial hydrodynamic coefficients and viscous hydrodynamic coefficients of the lander. The results show that the CFD can provide the hydrodynamic property for the moveable lander's design. The dynamic equations and kinematic equations are completely constructed combined with the hydrodynamic coefficients. Subsequently, this paper utilized the PID control method and $S$ control method to control the motions of the lander. The simulation results show that the methods accurately follow the preplanned path.
\end{abstract}

\section{Introduction}

At present, autonomous underwater vehicle (AUV) has been widely utilized in ocean exploration, marine science, marine sampling, and other fields [1]. Most of the existing AUVs work in shallow seas, such as for environmental mapping [2], the measurement of the synoptic observational field of current ocean surface radar [3], sample and data gathering [4-6], seabed inspection [7], and real-time water quality analysis [8]. The Lingyun moveable lander can provide assistance and guarantee for modular seabed landers operating at deep sea.

The hydrodynamic coefficient is significant to moveable lander design, motion control, and cruise plan [9]. The moveable lander is subjected to complex hydrodynamic conditions, strong coupling, and strong nonlinearity, since there are many influencing factors, such as shapes, current ocean conditions, and vehicle motion conditions. However, no similar-shape moveable lander has been applied to the deep sea, and let it alone dive down to the Mariana Trench. This study is based on this kind of situation. The methods of hydrodynamic coefficient analysis include the empirical method, system identification, free model test, captive model test, and computational fluid dynamic (CFD). The empirical method can quickly calculate the hydrodynamic coefficients, but its accuracy depends on the accumulation of experience and the shape of AUV [10,11]. Free model tests $[12,13]$ and captive model tests [14] can get many hydrodynamic coefficients directly, but these experiments are expensive, and the test equipment, such as large water tank and towing devices, is demanding, compared to the CFD method. Commonly, the captive model tests include a tow-tank experiment, rotating arm test, circular motion test, and planar motion mechanism (PMM). When we carry out a tow-tank test, the model is tilted into the pool by an angle (drift angle) concerning the direction of the drag, which can be used to measure velocity coefficients, et cetera. However, it is impossible to accurately measure the angular velocity coefficients and angular acceleration coefficients [15-18]. The rotating arm test makes it easy to measure individual rotate derivatives. However, the test equipment is large in scale and extremely expensive. The PMM test [19-21] can measure various 


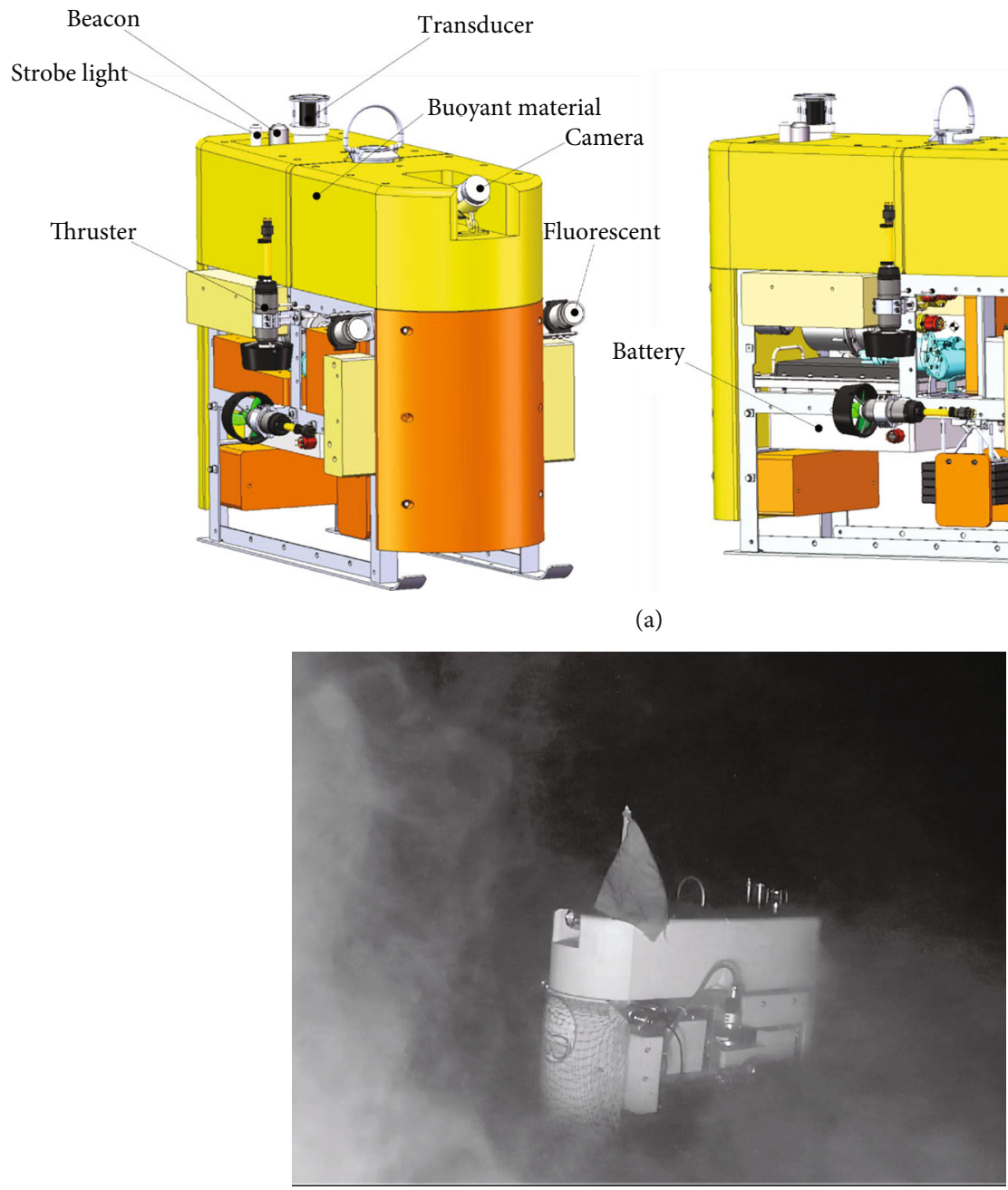

(b)

Figure 1: The Lingyun: (a) 3D model of the Lingyun; (b) Lingyun in the Mariana Trench.

Table 1: Parameters of the Lingyun.

\begin{tabular}{lccc}
\hline Parameter & Symbol & Unit & Value \\
\hline Length & $L$ & $\mathrm{~m}$ & 1.25 \\
Height & $H$ & $\mathrm{~m}$ & 0.93 \\
Width & $W$ & $\mathrm{~m}$ & 0.47 (excluding thrusters) \\
Superficial area & $A$ & $\mathrm{~m}^{2}$ & 4.411727 \\
Volume & $V$ & $\mathrm{~m}^{3}$ & 0.5066747 \\
Mass & $m$ & $\mathrm{~kg}$ & 519.798 \\
The moment of inertia of the $X$ axis & $I_{x}$ & $\mathrm{~kg} \cdot \mathrm{m}^{2}$ & 30.717279 \\
The moment of inertia of the $Y$ axis & $I_{y}$ & $\mathrm{~kg} \cdot \mathrm{m}^{2}$ & 47.148837 \\
The moment of inertia of the $Z$ axis & $I_{z}$ & $\mathrm{~kg} \cdot \mathrm{m}^{2}$ & 65.763013 \\
Diameter of the propeller & $D$ & $\mathrm{~mm}$ & 134
\end{tabular}

hydrodynamic coefficients, but it is limited by the length of the towing tank. Using finite element software to calculate the hydrodynamic coefficient is a practical, convenient, and low-cost method. Moreover, with the development of com- puter technology, the calculation result is getting faster in speed and better in precision. When we calculate the coefficient with the CFD method, the model needs to be accurately constructed. The next step is the meshing, followed 


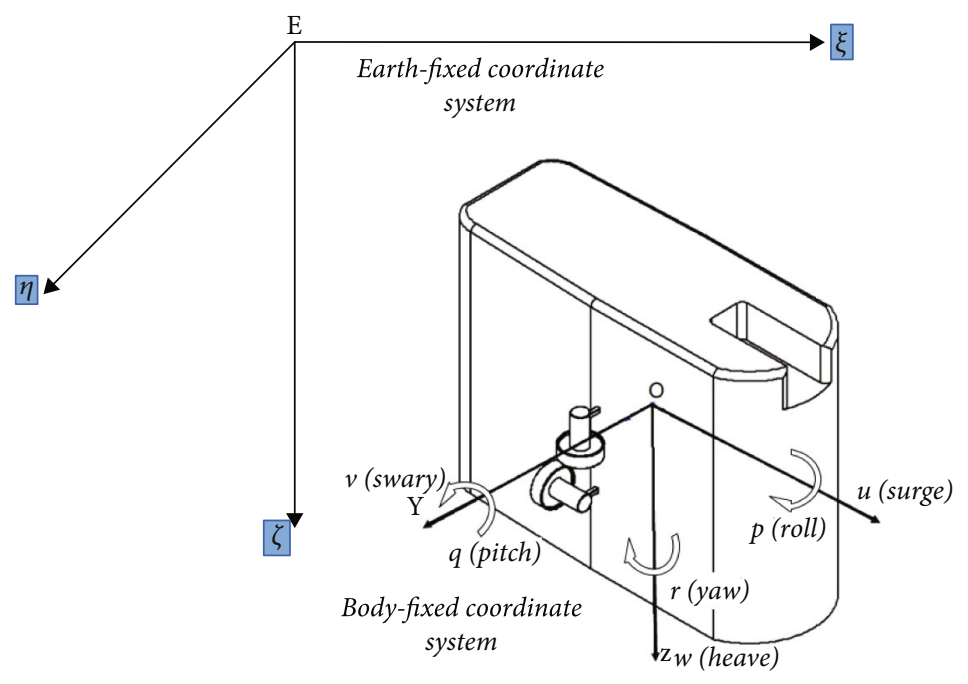

FIGURE 2: Frames and elementary lander motions.

by the boundary condition setting. The last step is the coefficient fitting [22]. In [23], the hydrodynamic coefficients of the TUNA-SAND AUV are calculated with the CFD method. Researchers analyze the influence of surface waves on the hydrodynamic performance of the AUV in [24]. The hydrodynamic coefficients of a moving body with NACA0012 hydrofoil utilizing CFD are calculated, and the relationship between oscillation frequency, amplitude, and hydrodynamic coefficients is discussed in [25].

In order to precisely control a moveable lander, its dynamic model needs to be established to meet hydrodynamic simulation and real-time control. As shown in Figure 1, the Lingyun, which is similar to the shape of the autonomous underwater vehicle in [26], is a four-degreeof-freedom moveable lander that can control the motion of surge, roll, heave, and yaw. The related parameters of the Lingyun moveable lander are shown in Table 1.

After calculating all the hydrodynamic coefficients, we establish the kinematic and dynamic model of the Lingyun lander. Then, the motion simulation is carried out by the PID control method and $S$ control method [27].

1.1. Kinematic and Dynamic Model. Kinematic and dynamic models are the theoretical basis for navigation and control. In order to establish the dynamic model of a movable lander, it is necessary to obtain its hydrodynamic characteristics and carry out experimental research on numerical simulation pools based on the research experience of AUV. Through the experimental design, the parameters of the experimental conditions are reasonably selected, the experimental data are fitted by the least squares, and the viscous hydrodynamic coefficients of the moveable lander are obtained.

The study of the motion of moveable landers generally uses two coordinate systems: body-fixed coordinate system (dynamic coordinate system) for studying the hydrodynamic characteristics and the inertial coordinate system (earth-fixed coordinate system) for describing the motion trajectory and position of the lander.
TABLE 2: Definition of dynamic parameters of a moveable lander.

\begin{tabular}{lcccccc}
\hline Axis & Position & Force & Moment & $\begin{array}{c}\text { Linear } \\
\text { velocity }\end{array}$ & $\begin{array}{c}\text { Angular } \\
\text { velocity }\end{array}$ & $\begin{array}{c}\text { Attitude } \\
\text { angle }\end{array}$ \\
\hline$X$ & $x$ & $X$ & $K$ & $u$ & $p$ & $\varphi$ \\
$\begin{array}{l}\text { axis } \\
Y\end{array}$ & $y$ & $Y$ & $M$ & $v$ & $q$ & $\theta$ \\
$\begin{array}{l}\text { axis } \\
Z\end{array}$ & $z$ & $Z$ & $N$ & $w$ & $r$ & $\psi$ \\
axis & & & & & & \\
\hline
\end{tabular}

Recommended by the International Towing Tank Conference (ITTC) and the Society of Naval Architects and Marine Engineers (SNAME), two coordinate systems are established, including the earth-fixed coordinate system and the body-fixed coordinate system, as shown in Figure 2. The inertial coordinate system $E-\xi \eta \psi$ is fixed to the earth. Origin $E$ is located at the sea surface or sea at a certain point; the $E \xi$ axis remains horizontal and takes the forward direction of the lander in the positive direction; the $E \eta$ axis is located on the horizontal plane where the $E \xi$ axis is located; according to the right-hand law, the axis rotates 90 degrees clockwise; the $E \zeta$ axis perpendicular to the $\xi E \eta$ plane in the center of the earth direction is positive. The body-fixed coordinate system $G-X Y Z$ is a right-angle coordinate system based on the lander's center of gravity $G$ as the coordinate origin, and it is fixed to the lander. The $X, Y$, and $Z$ axes are the intersections of horizontal, vertical, and midprofiles that pass through point $G$, respectively.

The linear velocity and angle velocity of the lander are represented by $u, v, w, p, q, r$. The accelerations and angular accelerations of the moveable lander are represented by $\dot{u}$, $\dot{v}, \dot{w}, \dot{p}, \dot{q}, \dot{r}$. Forces and moments are represented by $X, Y, Z$, $K, M, N$. The dynamic parameters of the moveable lander are defined as shown in Table 2:

The control position and attitude of the moveable lander can be determined by the earth-fixed coordinate values $\left(\xi_{0}\right.$, $\left.\eta_{0}, \zeta_{0}\right)$ of the origin of the body-fixed coordinate system and 
the attitude angles $(\varphi, \theta, \psi)$ of the body-fixed coordinate system (motion coordinate system) relative to the earth-fixed coordinate. $\varphi$ is the heeling angle, tilted to the right as a positive direction. $\theta$ is the trim angle, which indicates the positive direction when the stern is tilted. $\psi$ is the heading angle, where the right is turned positive.

The motion equation should be established in the earthfixed coordinate system and then converted to the bodyfixed coordinate system to derive the motion equation of a moveable lander under the body-fixed coordinate system.
The six-degree-of-freedom motion equation of a moveable lander is represented as the following vector form:

$$
\begin{aligned}
& \dot{\eta}=J(\eta) v, \\
& \eta=[\xi \eta \zeta \phi \theta \varphi]^{T}, \\
& v=\left[\begin{array}{llllll}
u & v & w & p & q & r
\end{array}\right]^{T},
\end{aligned}
$$

where

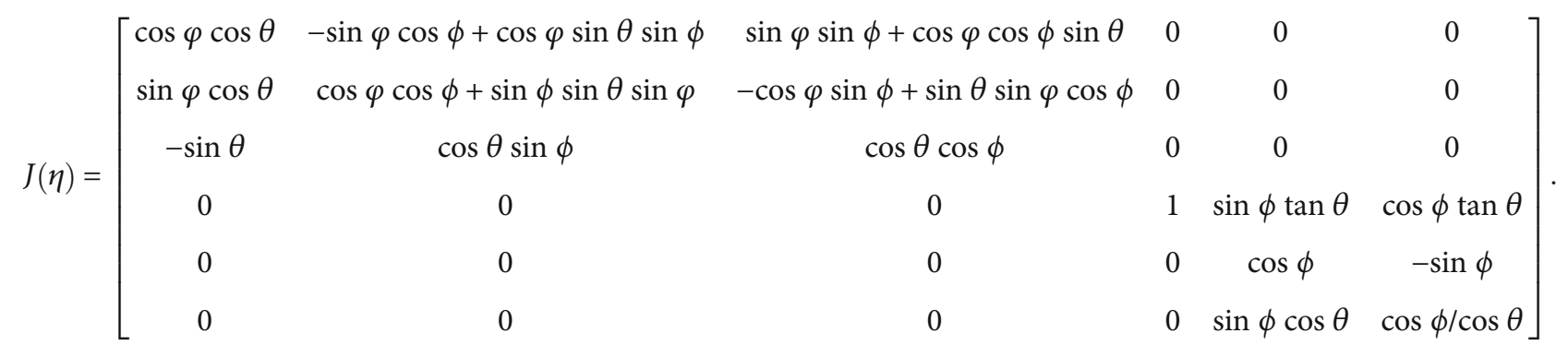

A moveable lander is regarded as a rigid body, based on the theoretical mechanics' speed synthesis theorem, and on the basis of the establishment of the coordinate system, the kinematic model of a moveable lander is introduced by momentum definition:

$$
\left\{\begin{array}{l}
m\left[(\dot{u}-v r+w q)-x_{G}\left(q^{2}+r^{2}\right)+y_{G}(p q-\dot{r})+z_{G}(p r+\dot{q})\right]=X, \\
m\left[(\dot{v}-w p+u r)-y_{G}\left(r^{2}+p^{2}\right)+z_{G}(q r-\dot{p})+x_{G}(q p+\dot{r})\right]=Y, \\
m\left[(\dot{w}-u q+v p)-z_{G}\left(p^{2}+q^{2}\right)+x_{G}(r p-\dot{q})+y_{G}(r q+\dot{p})\right]=Z, \\
I_{x} \dot{p}+\left(I_{z}-I_{y}\right) q r+m\left[y_{G}(\dot{w}+p v-q u)-z_{G}(\dot{v}+r u-p w)\right]=K, \\
I_{y} \dot{q}+\left(I_{x}-I_{z}\right) r p+m\left[z_{G}(\dot{u}+w q-v r)-x_{G}(\dot{w}+p v-u q)\right]=M, \\
I_{z} \dot{r}+\left(I_{y}-I_{x}\right) p q+m\left[x_{G}(\dot{v}+u r-p w)-y_{G}(\dot{u}+q w-v r)\right]=N,
\end{array}\right.
$$

where

$$
\left\{\begin{array}{l}
X=X_{I}+X_{\mathrm{vis}}+g_{X}+\tau_{X} \\
Y=Y_{I}+Y_{\mathrm{vis}}+g_{Y}+\tau_{Y} \\
Z=Z_{I}+Z_{\mathrm{vis}}+g_{Z}+\tau_{Z} \\
K=K_{I}+K_{\mathrm{vis}}+g_{K}+\tau_{K} \\
M=M_{I}+M_{\mathrm{vis}}+g_{M}+\tau_{M} \\
N=N_{I}+N_{\mathrm{vis}}+g_{N}+\tau_{N}
\end{array}\right.
$$

where $m$ is the mass of the moveable lander; $x_{G}, y_{G}, z_{G}$ are the coordinates of the center of gravities of the moveable lander; $I_{x}, I_{y}, I_{z}$ are the moments of inertia of the moveable lander's mass $m$ with respect to the $G X, G Y, G Z$ axes; $X_{I}$, $Y_{I}, Z_{I}, K_{I}, M_{I}, N_{I}$ are the inertial hydrodynamic forces and moments to which the moveable lander was subjected; $X_{\text {vis }}$ , $Y_{\text {vis }}, Z_{\text {vis }}, K_{\text {vis }}, M_{\text {vis }}, N_{\text {vis }}$ are the viscous hydrodynamic forces and moments to which the moveable lander was subjected; $\tau_{X}, \tau_{Y}, \tau_{Z}, \tau_{K}, \tau_{M}, \tau_{N}$ are the thrusts provided by the moveable lander thrusters; and $g_{X}, g_{Y}, g_{Z}, g_{K}, g_{M}, g_{N}$ are the static forces and moments.

1.2. The Analysis of Hydrodynamic Coefficients. There are two significances in studying hydrodynamic characteristics. On the one hand, the stability of a moveable lander is studied from the perspective of maneuverability. On the other hand, the influence of hydrodynamics needs to be considered in the design of the control system.

Generally speaking, the hydrodynamic characteristics and the hydrodynamic coefficients are related to the following factors of a moveable lander:

(1) The geometric shape of the moveable lander

(2) The motion state of the moveable lander referring to the motion parameters such as the linear velocity, angular velocity, and angular acceleration

(3) The property of the flow field, including the physical characteristics of the flow field and the geometric characteristics of the flow field

(4) Maneuver dynamical characteristics

In general, hydrodynamics is related to the characteristics of the moveable lander, the motion characteristics, and the fluid characteristics. If the structure of the moveable 
lander has been determined and is operating in an infinite stream field, hydrodynamic force $F_{F}$ is only related to the moveable lander's motion characteristics. It can be represented in the following form:

$$
F_{F}=f(V, \dot{V}, \Omega, \dot{\Omega})
$$

Equation (5) is a general expression of hydrodynamics.

Taylor's stage expansion is a valuable tool in studying hydrodynamic calculations. To simplify the problem, we assume that a moveable lander is moving in an infinite stream field, and the hydrodynamic effect is independent of its position in the flow field. Generally speaking, the hydrodynamics in its motion property is only related to the state of motion at that time, but not to the history of motion. Based on this assumption, only velocity and acceleration items are retained in hydrodynamic expansion, regardless of the derivatives of the second order and above of velocity to time.

Inertial hydrodynamic coefficients are generally obtained according to empirical methods. According to the theory of potential flow, the fluid inertial force is linearly related to acceleration, and considering the left and right symmetry of the Lingyun, the expression of inertial force acting on the Lingyun is as follows:

$$
\begin{aligned}
F_{I} & =\left[\begin{array}{llllll}
X_{I} & Y_{I} & Z_{I} & K_{I} & M_{I} & N_{I}
\end{array}\right]^{T} \\
& =R\left[\begin{array}{llllll}
\dot{u} & \dot{v} & \dot{w} & \dot{p} & \dot{q} & \dot{r}
\end{array}\right]^{T},
\end{aligned}
$$

where

$$
R=\left[\begin{array}{cccccc}
\frac{1}{2} \rho L^{3} X_{\dot{u}} & 0 & 0 & 0 & 0 & 0 \\
0 & \frac{1}{2} \rho L^{3} Y_{\dot{v}} & 0 & \frac{1}{2} \rho L^{4} K_{\dot{v}} & 0 & \frac{1}{2} \rho L^{4} N_{\dot{v}} \\
0 & 0 & \frac{1}{2} \rho L^{3} Z_{\dot{w}} & 0 & \frac{1}{2} \rho L^{4} M_{\dot{w}} & 0 \\
0 & \frac{1}{2} \rho L^{4} Y_{\dot{p}} & 0 & \frac{1}{2} \rho L^{5} K_{\dot{p}} & 0 & \frac{1}{2} \rho L^{5} N_{\dot{p}} \\
0 & 0 & \frac{1}{2} \rho L^{4} Z_{\dot{q}} & 0 & \frac{1}{2} \rho L^{5} M_{\dot{q}} & 0 \\
0 & \frac{1}{2} \rho L^{4} Y_{\dot{r}} & 0 & \frac{1}{2} \rho L^{5} K_{\dot{r}} & 0 & \frac{1}{2} \rho L^{5} N_{\dot{r}}
\end{array}\right],
$$

$$
\begin{gathered}
K_{11}=0.2 X_{\dot{u}}=-(\pi / 3)(B / L)(H / L) K_{11}=-5.8589 \times 10^{-2}, \\
K_{22}=1.3 Y_{\dot{v}}=-(\pi / 3)(B / L)(H / L) K_{22}=-0.38083, \\
K_{33}=0.4 Z_{\dot{w}}=-(\pi / 3)(B / L)(H / L) K_{33}=-0.11718, \\
K_{55}=0.8 M_{\dot{q}}=-(\pi / 60)(B / L)(H / L)\left(1+\left(B^{2} / L^{2}\right)\right) K_{55}=- \\
1.33745 \times 10^{-2}, \\
K 66=0.25 N_{\dot{r}}=-(\pi / 60)(B / L)(H / L)\left(1+\left(B^{2} / L^{2}\right)\right) K_{66}=
\end{gathered}
$$
$-5.6888 \times 10^{-3}$.

The viscous hydrodynamic coefficients of the moveable lander are related to its speed, i.e., $F_{\text {vis }}=f(u, v, w, p, q, r)$. In the Taylor expansion of viscous hydrodynamics, the hydrodynamic items related only to linear velocity $(u, v, w)$ are positional forces, whereas only the hydrodynamics related to angular velocity $(p, q, r)$ is rotational forces, and the others are coupled hydrodynamics. Positional forces are generally obtained by the wind tunnel test or tow-tank test, while rotational force and coupling hydrodynamic power are obtained mainly through the rotating arm test. When we select the CFD method to obtain the hydrodynamic coefficients, an appropriate virtual boundary is established to convert the bypass flow problem into an internal flow problem, and the RANS equation is utilized to solve in the space region formed by the virtual boundary and the moveable lander.

In this study, the CFD method is utilized to calculate the hydrodynamic coefficients, and the finite element software is used for simulation calculations. We mesh the lander in the virtual water tank at first in the preprocess software. The mesh is shown in Figure 3. In the simulation of the tow-tank test, the number of mesh grids obtained by the section is about 1.02 million. Moreover, in the simulation of the rotating arm test, the number of mesh grids obtained by the section is about 1.05 million. There are many settings in the preprocess, including setting grid boundaries, inlet, outlet, and other related parameters, such as setting dynamic viscosity to $0.001219 \mathrm{~kg} /(\mathrm{m} \cdot \mathrm{s})$, setting density to $1025.9 \mathrm{~kg} / \mathrm{m}^{3}$, setting molar mass to $18.02 \mathrm{~kg} / \mathrm{kmol}$, setting specific heat capacity to $0.932 \mathrm{~J} /(\mathrm{kg} \cdot \mathrm{K})$, setting the inlet speed to $1 \mathrm{knot}$, and setting the relative pressure of the outlet to $0 \mathrm{~Pa}$. The calculation and coefficient solution are based on the foregoing situation. Finally, in postprocessing, the calculated force and torque of 6 degrees of freedom, the velocity contour, the pressure 


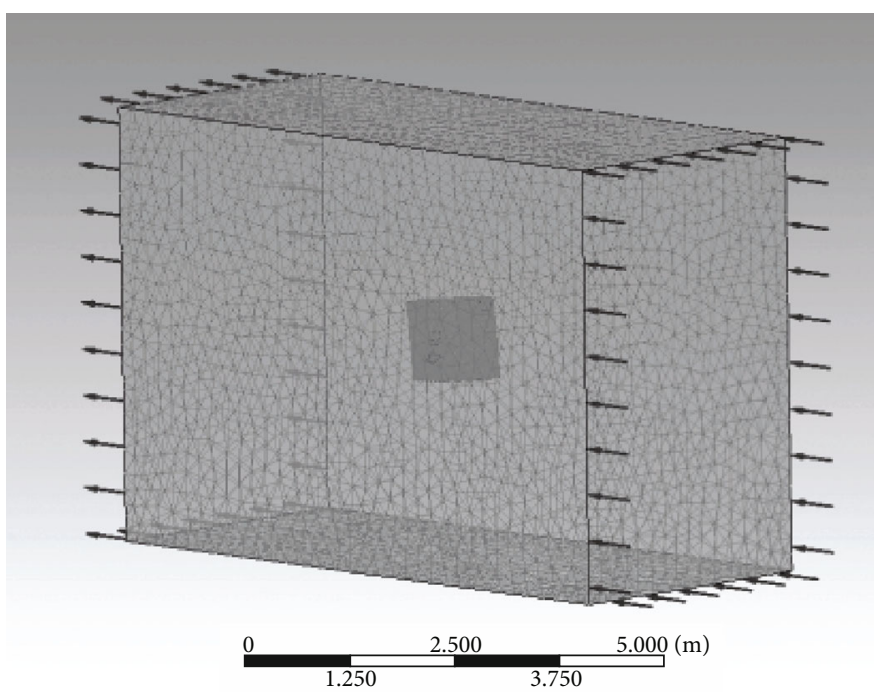

(a)

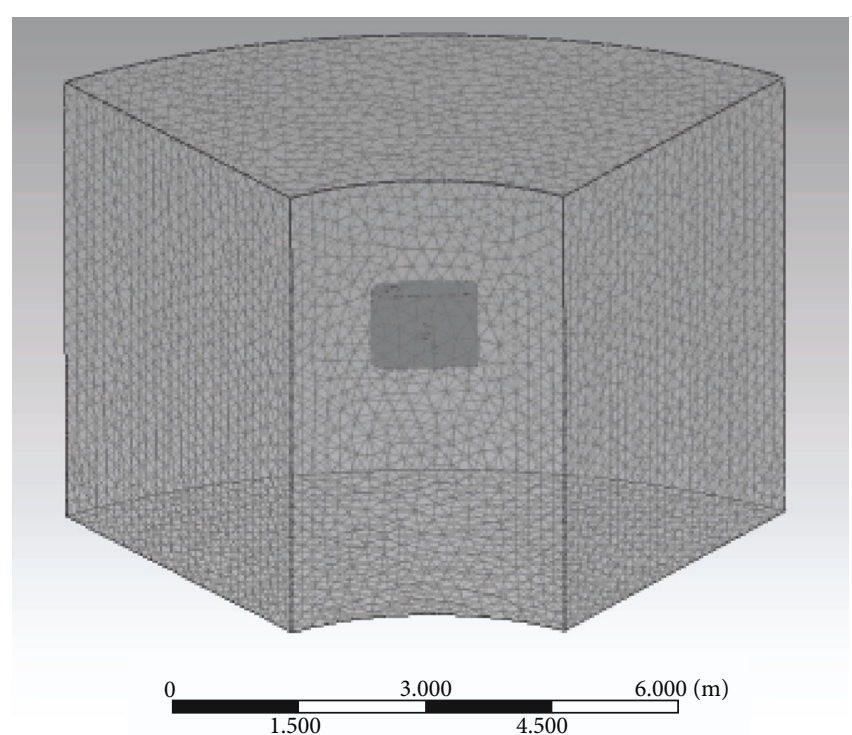

(b)

Figure 3: Mesh: (a) mesh for the tow-tank test; (b) mesh for the rotating arm test.

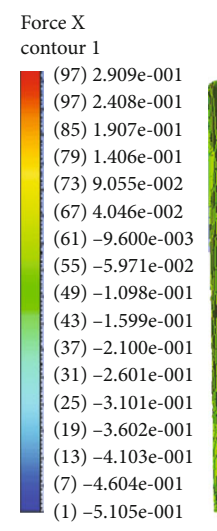

$(\mathrm{N})$
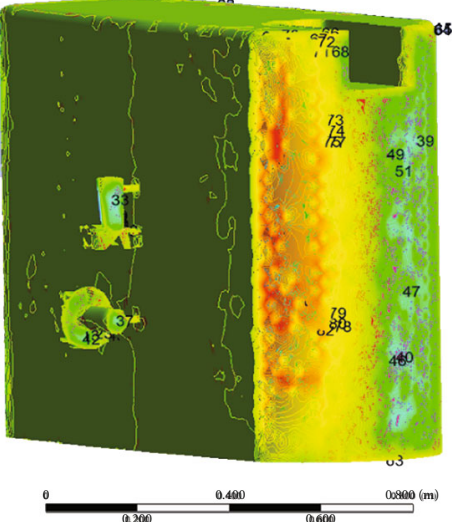

(a)
Force X

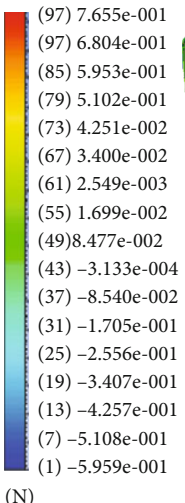

$(\mathrm{N})$
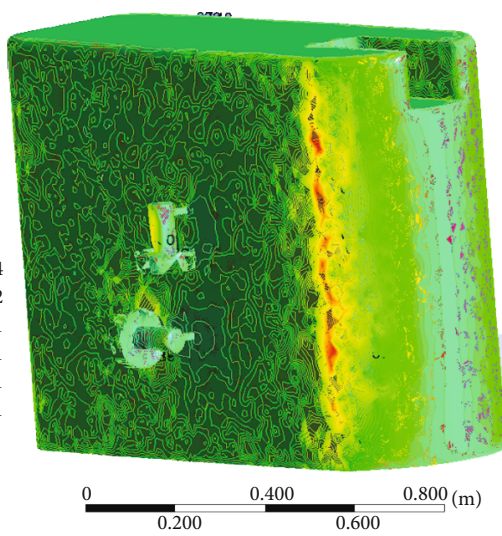

(b)

Figure 4: Viscous hydrodynamic contour: (a) force $X$ contour; (b) force $Y$ contour.

contour, the streamline, et cetera can be obtained, and the relevant results are shown in Figures 4-7.

The experiment is divided into three stages: the first stage calculates the navigational resistance of the moveable lander, the second stage is a single-plane test, and the third stage calculates the force in the coupling state and calculates the linear coupling derivative of hydrodynamics.

Positional forces are calculated using the $k-\varepsilon$ turbulence model, at the same inward flow speed $U$, the hydrodynamics with respect to different attack and drift angles are calculated, and the corresponding positional force coefficients are obtained by linear regression. In the simulation towtank test, the basin setting is shown in Figure 3(a): $5 \mathrm{~L}$ long, $6 W$ wide, and $6 D$ high ( $L, W$, and $D$ are the length, width, and height of the Lingyun, respectively). The vertical and moveable lander axis of the basin forms a certain angle. It means that the inward flow and the direction of the move- able lander movement form a certain angle of attack angle $\alpha$ or drift angle $\beta$.

For simulating the rotating arm test to calculate the rotational forces and coupled hydrodynamics of the moveable lander, the simulation computing environment is established and is shown in Figure 3(b), which is rotated by a rectangular circle of $6 \mathrm{~W} \times 6 \mathrm{H}$ around the center axis, and the arc length of the center arc of the fluid domain is $5 \mathrm{~L}$. The speed of the dynamic coordinate origin of the moveable lander is $U$, the angle of attack is $\alpha$, and the drift angle is $\beta$; then, the conversion matrix $T$ of the flow coordinate system to the body-fixed coordinate system is

$$
T=\left[\begin{array}{ccc}
\cos \alpha \cos \beta & \cos \alpha \sin \beta & -\sin \alpha \\
-\sin \beta & \cos \beta & 0 \\
\sin \alpha \cos \beta & \sin \alpha \sin \beta & \cos \alpha
\end{array}\right] .
$$


Force Z

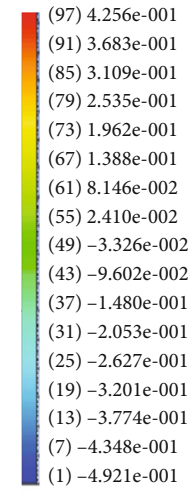

(N)

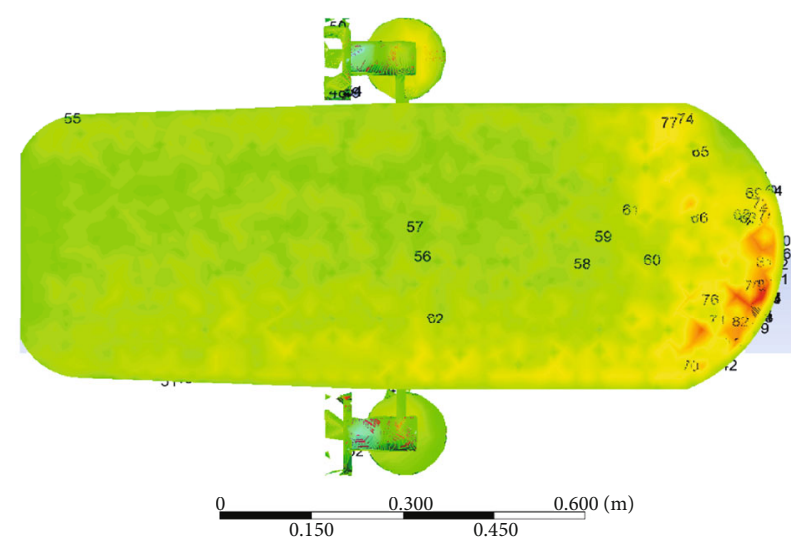

(a)

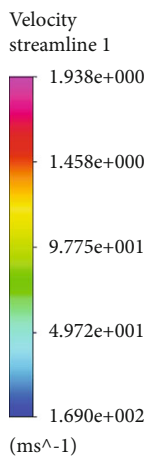

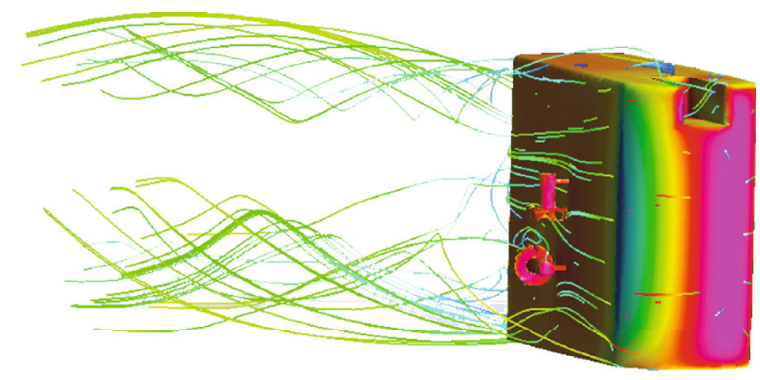

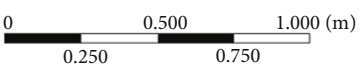

(b)

FIGURE 5: Contour and velocity streamline: (a) force $Z$ contour; (b) velocity stream line.

Pressure plane 1

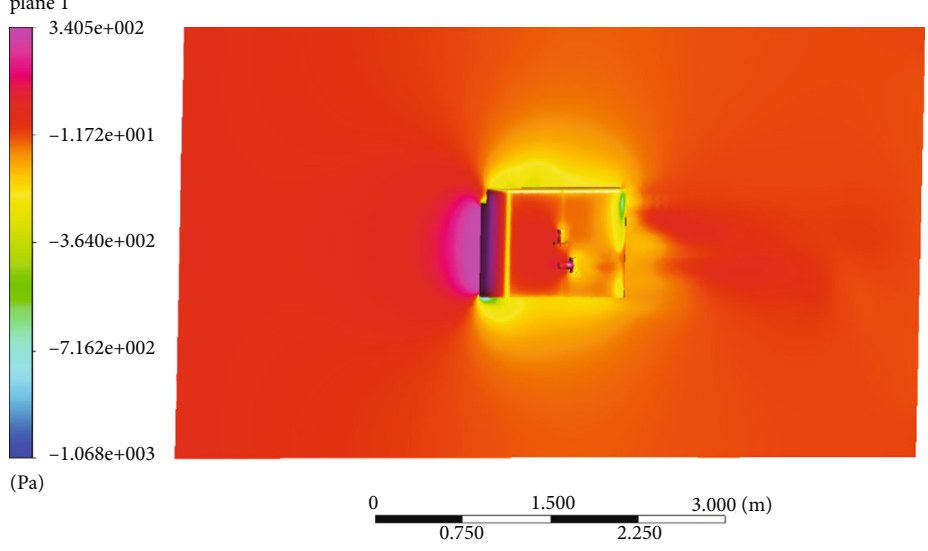

(a)

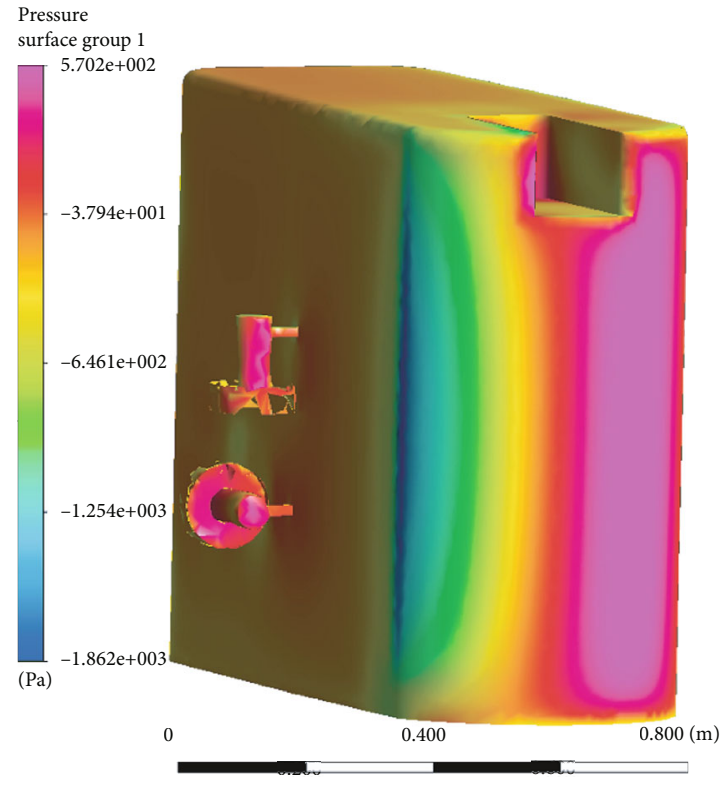

(b)

Figure 6: Pressure contour: (a) in entire simulation area; (b) in the Lingyun. 
Velocity

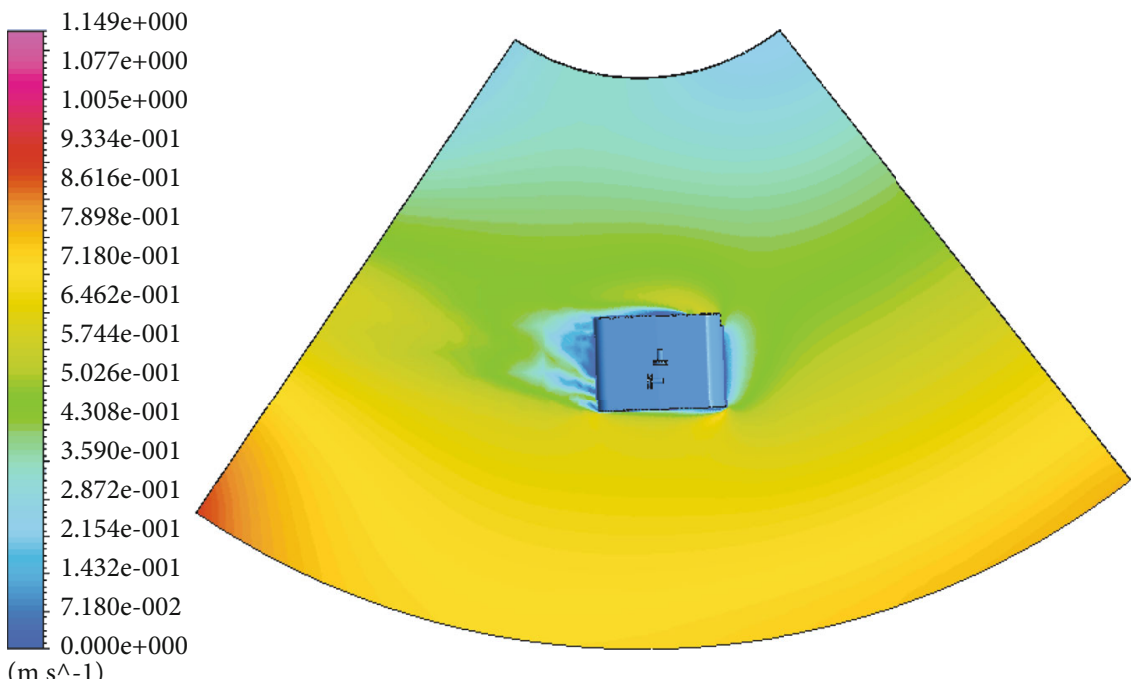

(a)

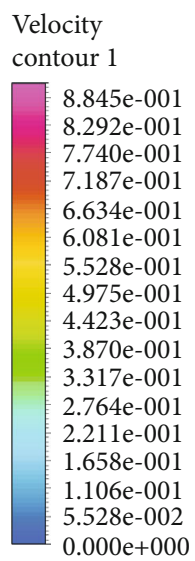

$\left(\mathrm{m} \mathrm{s}^{\wedge}-1\right)$

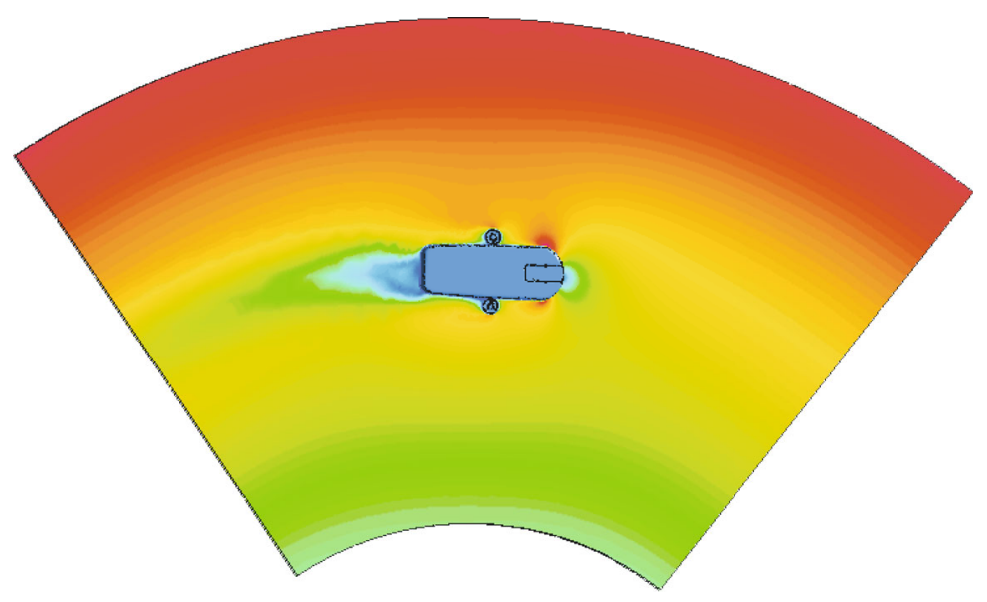

(b)

FIgURE 7: Velocity contour: (a) vertical plane; (b) horizontal plane.

TABLe 3: Calculation statistics table.

\begin{tabular}{|c|c|c|}
\hline Test items & Conditions & Numbers \\
\hline Drag calculation & $\begin{array}{c}U=0.25,0.5,0.75,1,1.25,1.5,1.75,2 \text { knots } \\
\alpha=\beta=0\end{array}$ & 8 \\
\hline Positional force calculation & $\begin{array}{l}U=1 \text { knot; } \beta=0, \alpha=-12,-11 \cdots \cdots 11,12 \\
U=1 \text { knot; } \alpha=0, \beta=-12,-11 \cdots \cdots 11,12\end{array}$ & 48 \\
\hline Coupled force calculation & $U=1 \mathrm{knot} ; \alpha=-3,3,6,9, \beta=-3,3,6,9$ & 16 \\
\hline Rotational force calculation (horizontal plane) & $\begin{array}{c}U=1 \mathrm{knot} ; R=5,10 \mathrm{~m} \\
\alpha=0, \beta=-3,0,3,6,9,12 \\
\beta=0, \alpha=-3,3,6,9,12\end{array}$ & 44 \\
\hline Rotational force calculation (vertical plane) & $\begin{array}{c}U=1 \mathrm{knot} ; R=5,10 \mathrm{~m} \\
\alpha=0, \beta=-3,0,3,6,9,12 \\
\beta=0, \alpha=-3,3,6,9,12\end{array}$ & 44 \\
\hline Total & & 160 \\
\hline
\end{tabular}


TABle 4: Simplified hydrodynamic coefficient.

\begin{tabular}{|c|c|c|c|c|c|}
\hline Coef & Value & Coef & Value & Coef & Value \\
\hline$X_{\dot{u}}$ & $-5.8589 e-02$ & $Y_{w p}$ & $1.0303904 e+01$ & $K_{p|p|}$ & $-2.80136 e+00$ \\
\hline$X_{u u}$ & $-1.35758 e-01$ & $Y_{p q}$ & $5.5370142 e+00$ & $K_{w p}$ & $7.22954 e-01$ \\
\hline$X_{v v}$ & $-8.89852 e-01$ & $Y_{w r}$ & $2.5674126 e+01$ & $K_{p q}$ & $2.16510 e-01$ \\
\hline$X_{w w}$ & $-1.00505 e+00$ & $Y_{q r}$ & $-4.9189501 e+01$ & $M_{\dot{q}}$ & $-1.33745 e-02$ \\
\hline$X_{q q}$ & $-1.82638 e+00$ & $Z_{\dot{w}}$ & $-1.1718 e-01$ & $M_{q|q|}$ & $1.33698 e-01$ \\
\hline$X_{r r}$ & $1.15448 e+00$ & $Z_{q|q|}$ & $-2.3971221 e+00$ & $M_{p p}$ & $-8.35750 e-01$ \\
\hline$X_{p r}$ & $-2.50100 e+00$ & $Z_{w w}$ & $1.4742334 e+00$ & $N_{\dot{r}}$ & $-5.6888 e-03$ \\
\hline$Y_{\dot{v}}$ & $-3.8083 e-01$ & $Z_{p p}$ & $4.8484061 e+00$ & $N_{p|p|}$ & $-8.41556 e+00$ \\
\hline$Y_{p}$ & $3.70578 e+04$ & $Z_{w \mid \sqrt{v^{2}+w^{2}}} \mid$ & $-1.3851717 e+00$ & $N_{v w}$ & $1.00346 e-01$ \\
\hline$Y_{p|p|}$ & $-8.47231 e+00$ & $Z_{w q}$ & $1.5969039 e-01$ & $N_{(v /|v|) \mid} \sqrt{v^{2}+w^{2}}|| r \mid$ & $3.95702 e-02$ \\
\hline$Y_{v|v|}$ & $-7.85474 e-01$ & $Z_{v p}$ & $1.1612743 e+00$ & $N_{v q}$ & $-5.01860 e-01$ \\
\hline$Y_{|v| v}$ & $-1.00552 e+00$ & $Z_{p r}$ & $1.0412445 e+00$ & $N_{w p}$ & $1.63202 e+00$ \\
\hline$Y_{v q}$ & $2.35154 e+01$ & $Z_{|w|}$ & $-6.6430789 e+00$ & $N_{w r}$ & $-1.64769 e-01$ \\
\hline
\end{tabular}

When the moveable lander rotates around the $\zeta$-axis of the earth-fixed coordinate system at an angular velocity $\omega$, the components $(p, q, r)$ of the angular velocity in the body-fixed coordinate system are

$$
\left[\begin{array}{l}
p \\
q \\
r
\end{array}\right]=T\left[\begin{array}{l}
0 \\
0 \\
\omega
\end{array}\right]=\left[\begin{array}{c}
-\omega \sin \alpha \\
0 \\
\omega \cos \alpha
\end{array}\right] .
$$

The situation is similar when the moveable lander rotates around the $x$-axis and $y$-axis at the angular velocity $\omega$.

Viscous hydrodynamics includes the linear, nonlinear, and coupling hydrodynamics caused by the interaction of horizontal and vertical surface motion. The highest order is taken as second order, and the conditions of all computational simulations in this study are shown in Table 3.

The six-degree-of-freedom viscous hydrodynamics is shown as formulas (10), (11), (12), (13), (14), and (15). The simplified hydrodynamic coefficients on the six degrees of freedom fitted from the calculations results are shown in Table 4 (inertial hydrodynamic coefficients are included in the table). The viscous hydrodynamic coefficients are all dimensionless numbers, and for the viscous hydrodynamic coefficients, the dimensionless coefficient to $u^{2}, v^{2}, w^{2}$ is $1 / 2$ $\rho L^{2}$, the dimensionless coefficient to $q^{2}, r^{2}, r p$ is $1 / 2 \rho L^{4}$, and the dimensionless coefficient to $v r, w q$ is $1 / 2 \rho L^{3}$, and for the viscous hydrodynamic moment coefficients, the dimensionless coefficient to $u^{2}, v^{2}, w^{2}$ is $1 / 2 \rho L^{3}$, the dimensionless coefficient to $q^{2}, r^{2}, r p$ is $1 / 2 \rho L^{5}$, and the dimensionless coefficient to $v r, w q$ is $1 / 2 \rho L^{4}$. The relationship between angular velocity and forces (moments) in the rotating arm test is shown in Figure 8. The relationship between drag and velocity is shown in Figure 9

$$
\begin{aligned}
& X_{\mathrm{vis}}=X_{u u} u^{2}+X_{v v} v^{2}+X_{w w} w^{2}+X_{r r} r^{2}+X_{q q} q^{2} \\
& +X_{v r} v r+X_{w q} w q+X_{p r} p r \\
& Y_{\mathrm{vis}}=Y_{v} v+Y_{r} r+Y_{r|r|} r|r|+Y_{p} p+Y_{p|p|} p|p| \\
& +Y_{v w} v w+Y_{|v| v} v\left|\sqrt{v^{2}+w^{2}}\right| \\
& +Y_{v|v|} \frac{v}{|v|}\left|\sqrt{v^{2}+w^{2}}\right||v|+Y_{v q} v q+Y_{w p} w p \\
& +Y_{p q} p q+Y_{w r} w r+Y_{q r} q r+Y_{w} w \\
& Z_{\mathrm{vis}}=Z_{w} w+Z_{q} q+Z_{q|q|} q|q|+Z_{w w} w^{2}+Z_{v v} v^{2} \\
& +Z_{r r} r^{2}+Z_{p p} p^{2}+Z_{w|w|} w|w| \\
& +Z_{w \mid \sqrt{v^{2}+w^{2}}} w\left|\sqrt{v^{2}+w^{2}}\right| \\
& +Z_{\frac{w}{|w|}\left|\sqrt{v^{2}+w^{2}}\right||q|} \frac{w}{|w|}\left|\sqrt{v^{2}+w^{2}}\right||q| \\
& +Z_{w q} w q+Z_{v p} v p+Z_{p r} p r+Z_{v r} v r+Z_{|w|}|w| \text {, } \\
& K_{\mathrm{vis}}=K_{u} u+K_{v} v+K_{w} w+K_{q} q+K_{u u} u^{2}+K_{r r} r^{2} \\
& +K_{p|p|} p|p|+K_{v|v|} v|v|+K_{v q} v q+K_{w p} w p \\
& +K_{w r} w r+K_{p q} p q+K_{u v} u v+K_{q q} q^{2} \text {, } \\
& M_{\mathrm{vis}}=M_{w} w+M_{q} q+M_{q|q|} q|q|+M_{r r} r^{2}+M_{p p} p^{2} \\
& +M_{w\left|\sqrt{v^{2}+w^{2}}\right|} w\left|\sqrt{v^{2}+w^{2}}\right| \\
& +M_{\frac{w}{|w|} \mid \sqrt{v^{2}+w^{2}}}|r| \frac{w}{|w|}\left|\sqrt{v^{2}+w^{2}}\right||r| \\
& +M_{v r} v r+M_{u} u+M_{v} v \text {, }
\end{aligned}
$$



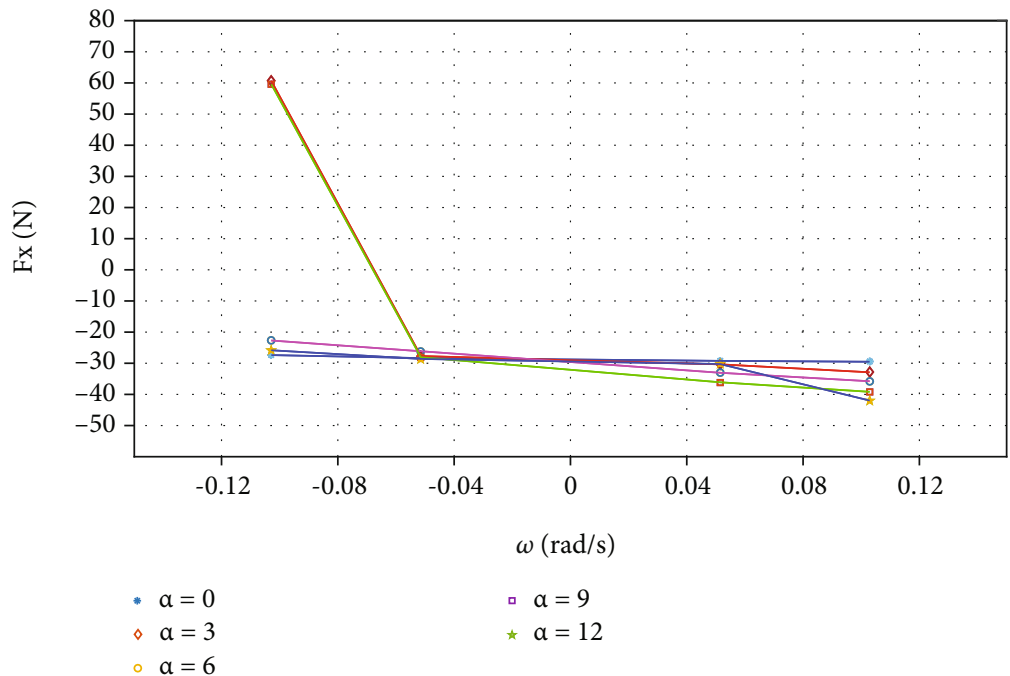

(a)

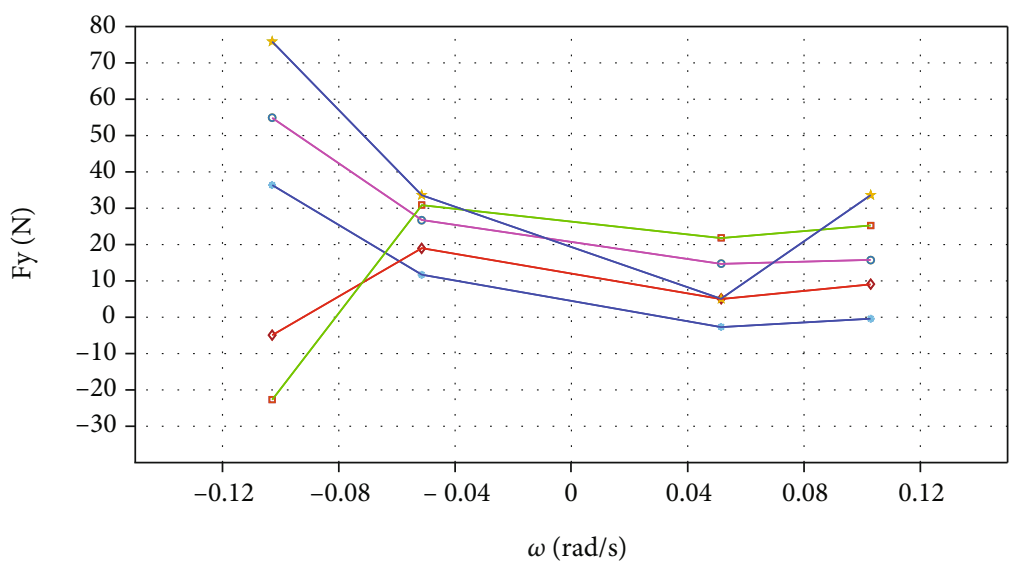
- $a=0$
- $\alpha=9$
- $a=3$
$\star \alpha=12$

(b)

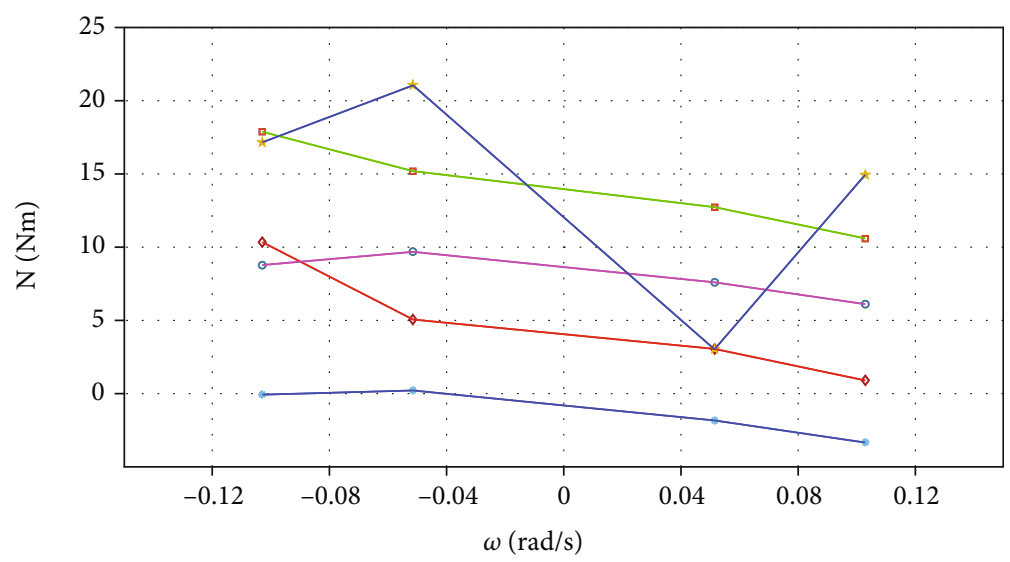
* $a=0$
$\diamond \alpha=3$
- $a=9$
* $a=12$

- $\alpha=6$

(c)

Figure 8: Continued. 


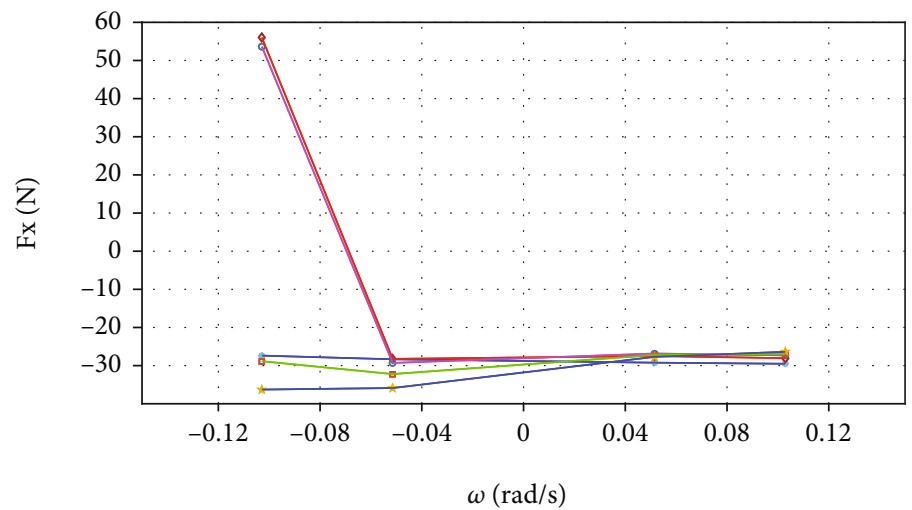

$$
\begin{array}{ll}
\text { - } \beta=0 & \text { ॰ } \beta=9 \\
\text { ॰ } \beta=3 & \text { * } \beta=12 \\
\text { } \beta=6 &
\end{array}
$$

(d)

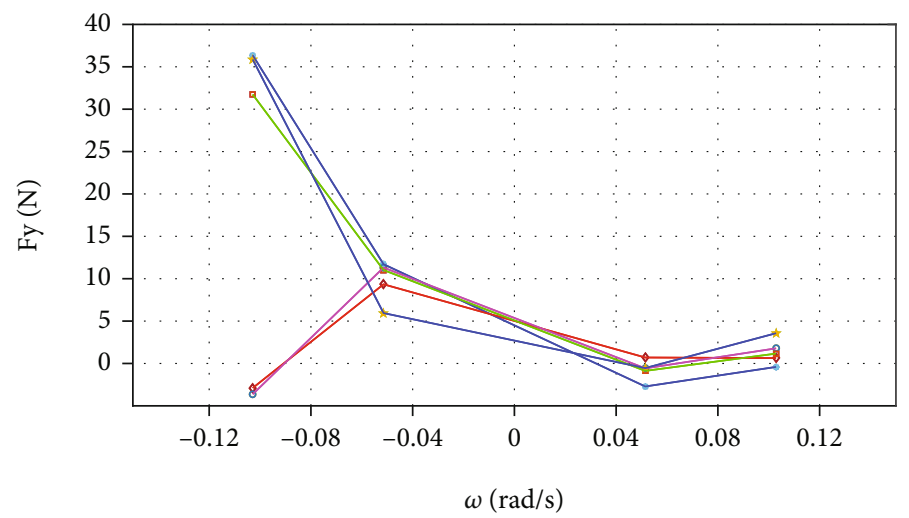
- $\beta=0$
- $\beta=9$
- $\beta=3$
$\star \beta=12$
- $\beta=6$

(e)

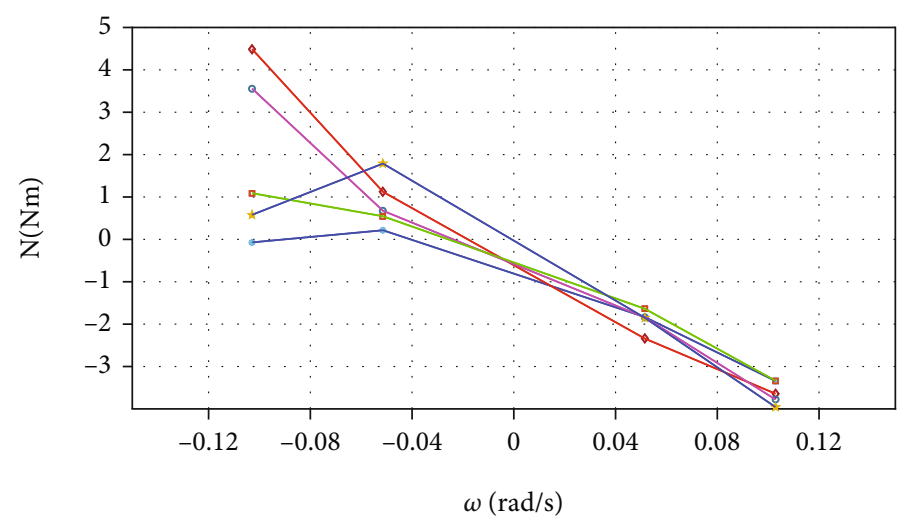
- $\beta=0$
- $\beta=9$
- $\beta=3$
$\star \beta=12$
- $\beta=6$

(f)

FIgUre 8: Continued. 


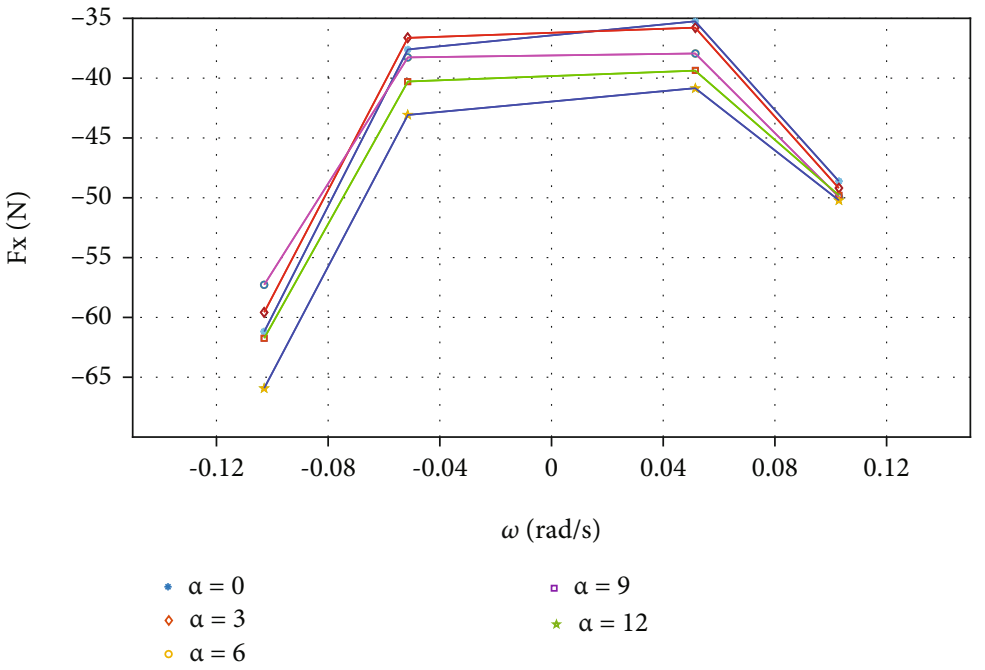

(g)
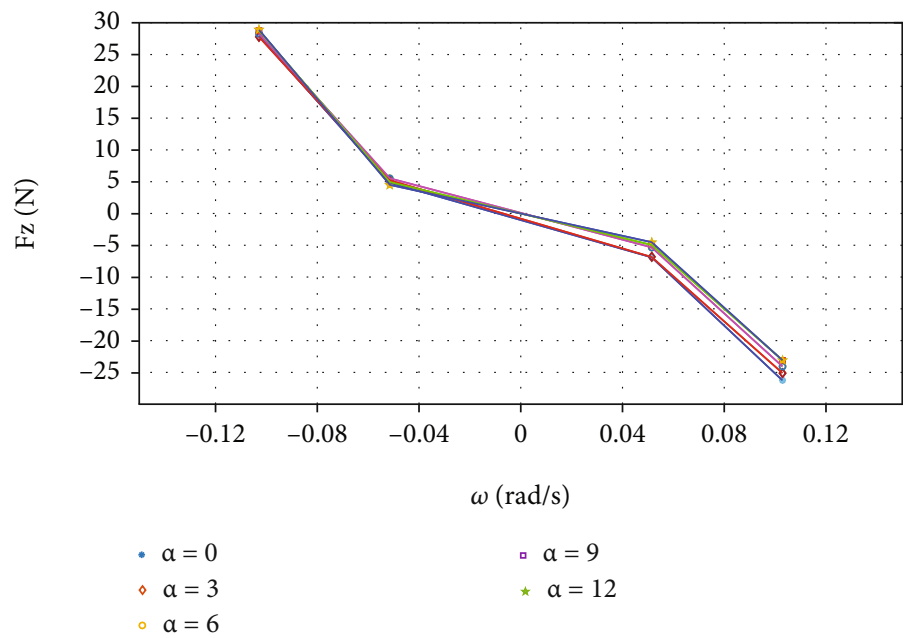

(h)

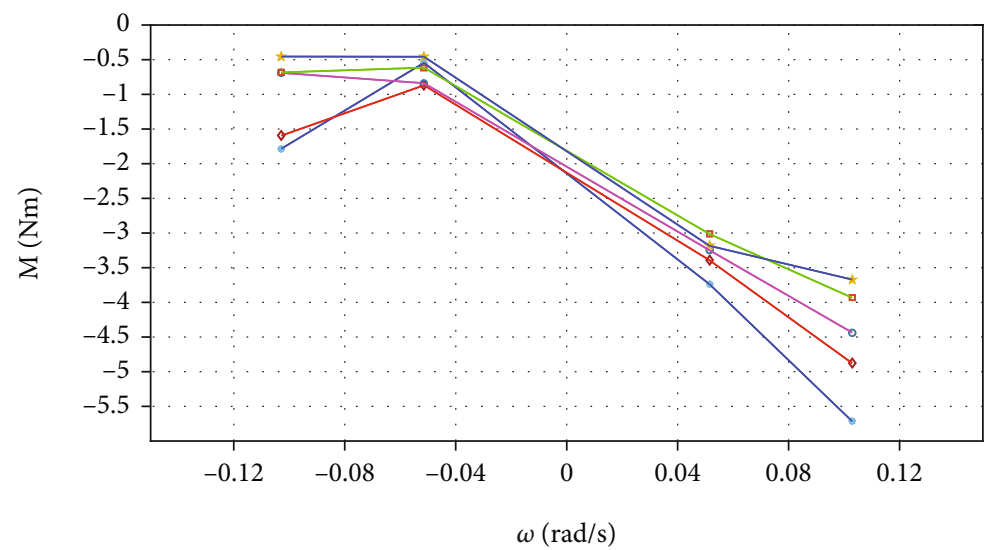

$$
\begin{aligned}
& \text { - } a=0 \\
& \text { - } a=3
\end{aligned}
$$$$
\text { - } a=9
$$$$
\text { * } a=12
$$

(i)

Figure 8: Continued. 


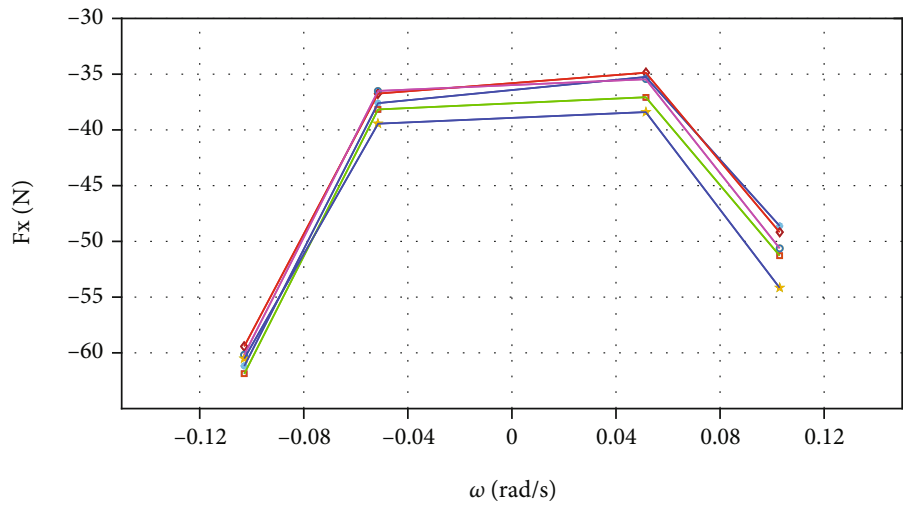

$$
\begin{array}{ll}
* \beta=0 & \square \beta=9 \\
\diamond \beta=3 & \star \beta=12 \\
\circ \beta=6 &
\end{array}
$$

(j)

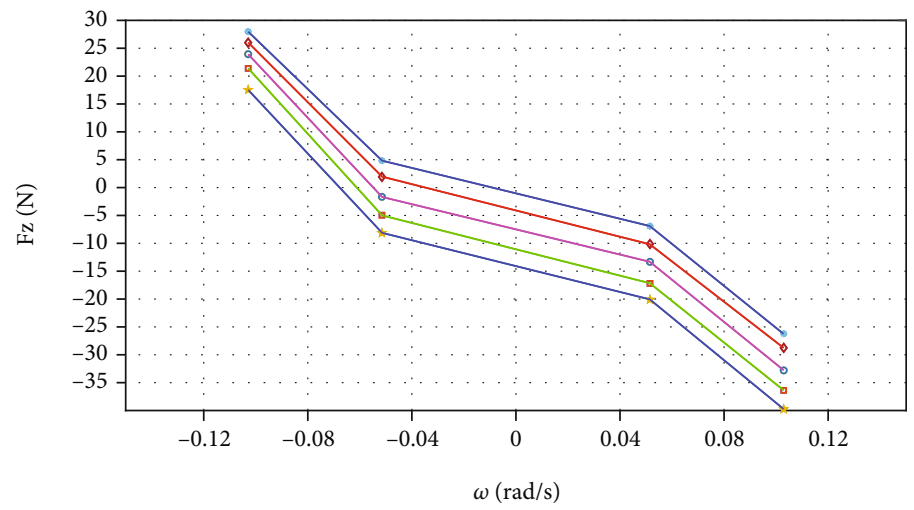

$$
\begin{array}{ll}
* \beta=0 & \square \beta=9 \\
\circ \beta=3 & * \beta=12 \\
\circ \beta=6 &
\end{array}
$$

$(\mathrm{k})$

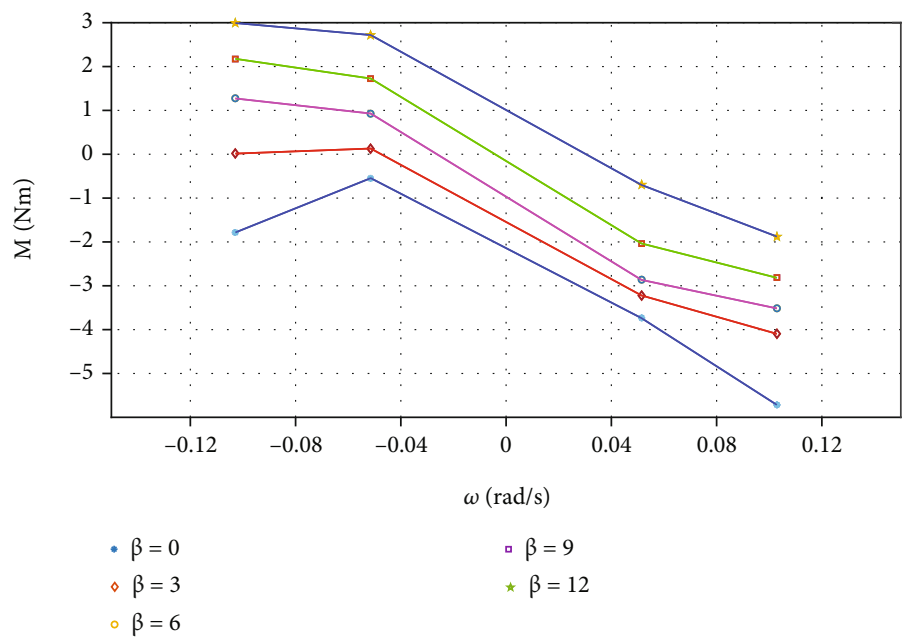

(1)

Figure 8: Relationship between angular velocity and forces and moments in the rotating arm test: (a) between $F x$ and $\omega$ ( $\beta=0)$ in the horizontal plane; (b) between $F y$ and $\omega(\beta=0)$; (c) between $N$ and $\omega(\beta=0)$; (d) between $F x$ and $\omega(\alpha=0)$ in the horizontal plane; (e) between $F y$ and $\omega(\alpha=0)$; (f) between $N$ and $\omega(\alpha=0)$; (g) between $F x$ and $\omega(\beta=0)$ in the vertical plane; (h) between Fz and $\omega(\beta=0)$; (i) between $M$ and $\omega(\beta=0)$; (j) between $F x$ and $\omega(\alpha=0)$ in the vertical plane; (k) between $F z$ and $\omega(\alpha=0)$; (l) between $M$ and $\omega(\alpha=0)$. 


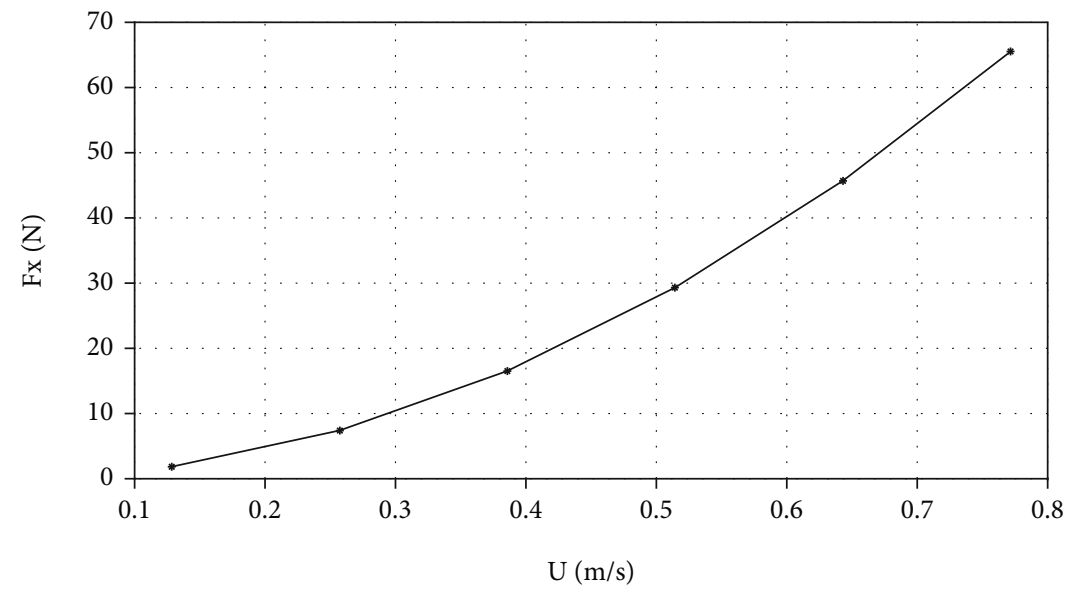

FIGURE 9: Relationship between the drag force and velocity.

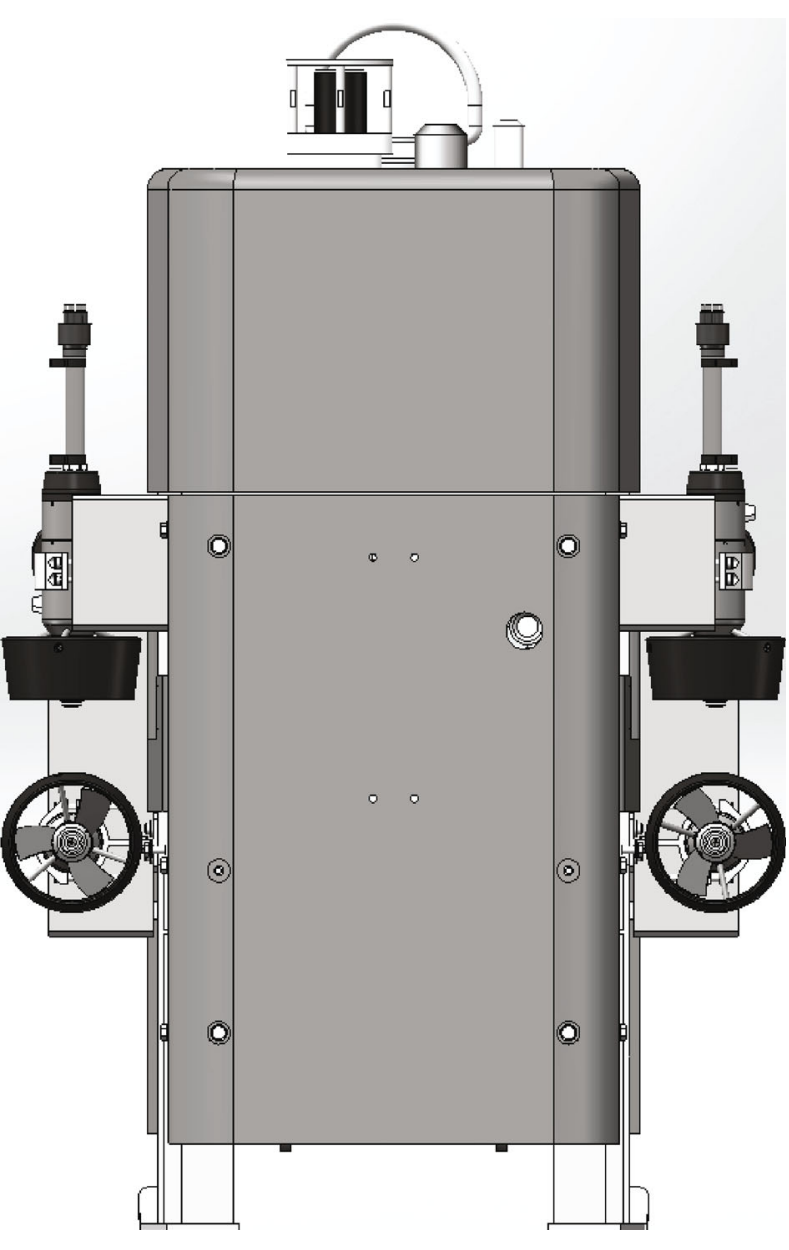

(a)

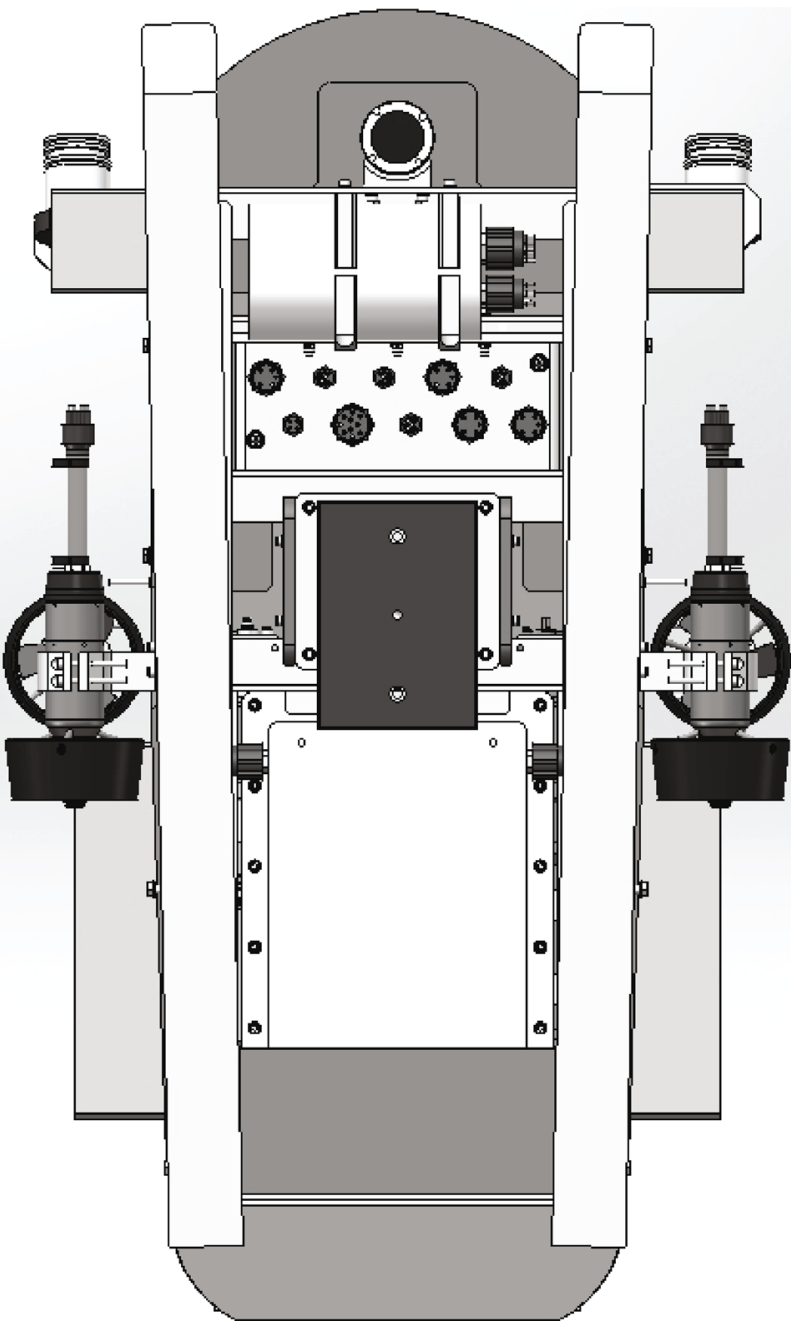

(b)

FIGURE 10: Diagram of thruster configuration: (a) rear view; (b) top view. 


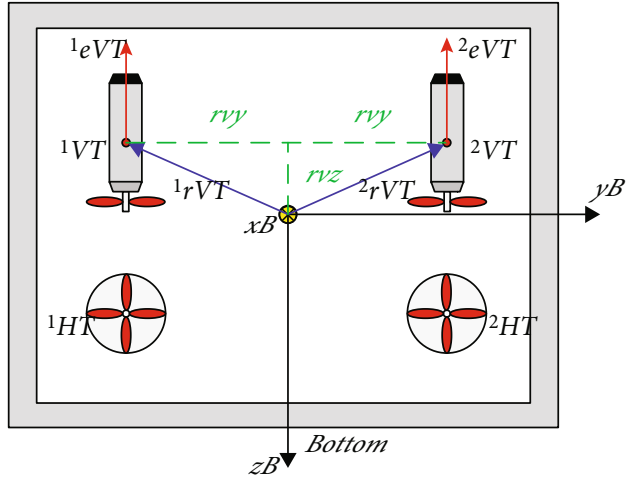

(a)

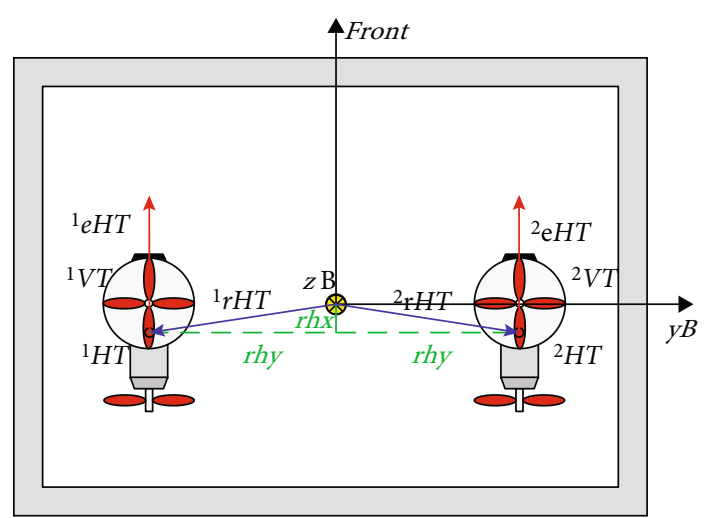

(b)

FIgURE 11: Diagram of thruster configuration: (a) rear view; (b) top view.

TABle 5: Position vectors for thruster configuration of the Lingyun.

\begin{tabular}{lccc}
\hline \multicolumn{2}{c}{ Horizontal thrusters } & \multicolumn{2}{c}{ Vertical thrusters } \\
${ }^{1} r_{\mathrm{HT}}$ & ${ }^{2} r_{\mathrm{HT}}$ & ${ }^{1} r_{\mathrm{VT}}$ & ${ }^{2} r_{\mathrm{VT}}$ \\
\hline$\left[\begin{array}{c}-r_{h x} \\
-r_{h y} \\
0\end{array}\right]$ & {$\left[\begin{array}{c}0 \\
-r_{h x} \\
r_{h y} \\
0\end{array}\right]$} & {$\left[\begin{array}{c}0 \\
r_{v y} \\
-r_{v z}\end{array}\right]$} & {$\left[\begin{array}{c}r_{v y} \\
-r_{v z}\end{array}\right]$} \\
\hline
\end{tabular}

$$
\begin{aligned}
N_{\text {vis }}= & N_{v} v+N_{r} r+N_{r|r|} r|r|+N_{p}|p|+N_{p|p|} p|p| \\
& +N_{v w} v w+N_{v\left|\sqrt{v^{2}+w^{2}}\right|} v\left|\sqrt{v^{2}+w^{2}}\right| \\
& +N_{\frac{v}{\mid v} \mid \sqrt{v^{2}+w^{2}}}|| r\left|\frac{v}{|v|}\right| \sqrt{v^{2}+w^{2}}|| r \mid+N_{v q} v q \\
& +N_{w p} w p+N_{p q} p q+N_{w r} w r+N_{w} w .
\end{aligned}
$$

According to equations (3), (10), (11), (12), (13), (14), and (15), the simplified six-degree-of-freedom space motion equations of the Lingyun are as follows:

Surge motion equation:

$$
\begin{aligned}
& m\left[\dot{u}-v r+w q-x_{G}\left(q^{2}+r^{2}\right)+y_{G}(p q-\dot{r})+z_{G}(p r+\dot{q})\right] \\
& =\frac{1}{2} \rho L^{4}\left[X_{q q}^{\prime} q^{2}+X_{r r}^{\prime} r^{2}+X_{p r}^{\prime} p r\right]+\frac{1}{2} \rho L^{3} X_{\dot{u}} \dot{u} \\
& \quad+\frac{1}{2} \rho L^{2}\left[X_{u u}^{\prime} u^{2}+X_{v v}^{\prime} v^{2}+X_{w w}^{\prime} w^{2}\right] \\
& \quad-(W-B) \sin \theta+\tau_{X} .
\end{aligned}
$$

Sway motion equation:

$$
\begin{aligned}
m[\dot{v} & \left.-w p+u r-y_{G}\left(r^{2}+p^{2}\right)+z_{G}(q r-\dot{p})+x_{G}(p q+\dot{r})\right] \\
= & Y_{p} p+\frac{1}{2} \rho L^{4}\left[Y_{p|p|} p|p|+Y_{p q} p q+Y_{q r} q r\right] \\
& +\frac{1}{2} \rho L^{3}\left[Y_{\dot{v}} \dot{v}+Y_{v q} v q+Y_{w p} w p+Y_{w r} w r\right] \\
& +(W-B) \cos \theta \sin \varphi+\tau_{Y} \\
& +\frac{1}{2} \rho L^{2}\left[Y_{v|v|} \frac{v}{|v|}\left|\sqrt{v^{2}+w^{2}}\right||v|+Y_{|v| v} v\left|\sqrt{v^{2}+w^{2}}\right|\right] .
\end{aligned}
$$

TABLE 6: Orientation vectors for thruster configuration of the Lingyun.

\begin{tabular}{lccc}
\hline \multicolumn{2}{c}{ Horizontal thrusters } & \multicolumn{2}{c}{ Vertical thrusters } \\
${ }^{1} e_{\mathrm{HT}}$ & ${ }^{2} e_{\mathrm{HT}}$ & ${ }^{1} e_{\mathrm{VT}}$ & ${ }^{2} e_{\mathrm{VT}}$ \\
\hline$\left[\begin{array}{l}1 \\
0 \\
0\end{array}\right]$ & {$\left[\begin{array}{l}1 \\
0 \\
0\end{array}\right]$} & {$\left[\begin{array}{c}0 \\
0 \\
-1\end{array}\right]$} & {$\left[\begin{array}{c}0 \\
0 \\
-1\end{array}\right]$} \\
\hline
\end{tabular}

Heave motion equation:

$$
\begin{aligned}
m[\dot{w} & \left.-u q+v p-z_{G}\left(p^{2}+q^{2}\right)+x_{G}(r p-\dot{q})+y_{G}(r q+\dot{p})\right] \\
= & Z_{|w|}|w|+\frac{1}{2} \rho L^{4}\left[Z_{p p} p^{2}+Z_{p r} p r+Z_{q|q|} q|q|\right] \\
& +\frac{1}{2} \rho L^{3}\left[Z_{\dot{w}} \dot{w}+Z_{v p} v p+Z_{w q} w q\right] \\
& +\frac{1}{2} \rho L^{2}\left[Z_{w w} w^{2}+Z_{w \mid \sqrt{v^{2}+w^{2}}} w\left|\sqrt{v^{2}+w^{2}}\right|\right] \\
& +(W-B) \cos \theta \cos \varphi+\tau_{Z} .
\end{aligned}
$$

Roll motion equation:

$$
\begin{aligned}
I_{x} \dot{p}+ & \left(I_{z}-I_{y}\right) q r+m\left[y_{G}(\dot{w}-u q+v p)-z_{G}(\dot{v}-w p+u r)\right] \\
= & \frac{1}{2} \rho L^{5}\left[K_{p|p|} p|p|+K_{p q} p q\right]+\frac{1}{2} \rho L^{4} K_{w p} w p \\
& +\left(y_{G} W-y_{C} B\right) \cos \theta \cos \varphi \\
& -\left(z_{G} W-z_{C} B\right) \cos \theta \sin \varphi+\tau_{K} .
\end{aligned}
$$

Pitch motion equation:

$$
\begin{aligned}
I_{y} \dot{q}+ & \left(I_{x}-I_{z}\right) r p+m\left[z_{G}(\dot{u}-v r+w q)-x_{G}(\dot{w}-u q+v p)\right] \\
= & \frac{1}{2} \rho L^{5}\left[M_{\dot{q}} \dot{q}+M_{p p} p^{2}+M_{q|q|} q|q|\right] \\
& +\left(x_{G} W-x_{C} B\right) \cos \theta \cos \varphi \\
& -\left(z_{G} W-z_{C} B\right) \sin \theta+\tau_{M} .
\end{aligned}
$$




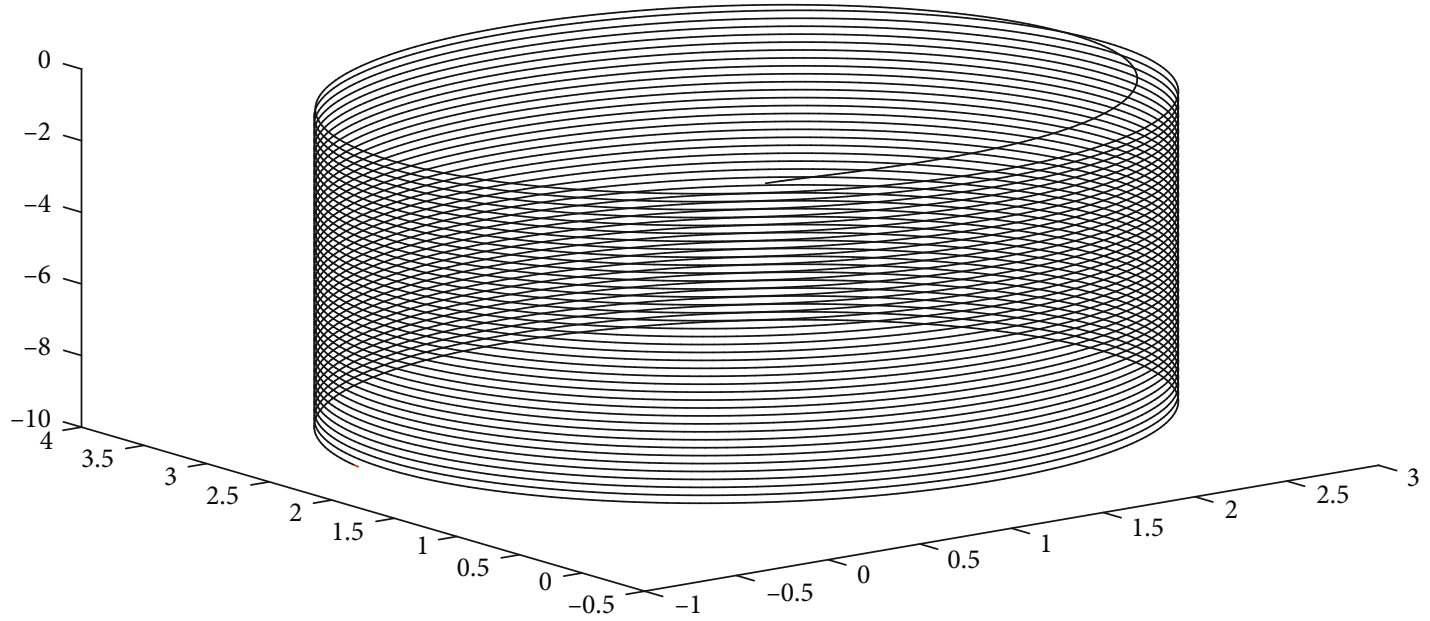

Figure 12: 3D motion simulation.

Yaw motion equation:

$$
\begin{aligned}
I_{z} \dot{r}+ & \left(I_{y}-I_{x}\right) p q+m\left[x_{G}(\dot{v}-w p+u r)-y_{G}(\dot{u}-v r+w q)\right] \\
= & \frac{1}{2} \rho L^{5}\left[N_{\dot{r}} \dot{r}+N_{p|p|} p|p|\right]+\frac{1}{2} \rho L^{3}\left[N_{v w} v w\right] \\
& +\frac{1}{2} \rho L^{4}\left[N_{w r} w r+N_{w p} w p+N_{v q} v q\right. \\
& \left.+N_{|v|}\left|\sqrt{v^{2}+w^{2}}\right||r| \frac{v}{|v|}\left|\sqrt{v^{2}+w^{2}}\right||r|\right] \\
& +\left(x_{G} W-x_{C} B\right) \cos \theta \sin \varphi \\
& +\left(y_{G} W-y_{C} B\right) \sin \theta+\tau_{N} .
\end{aligned}
$$

1.3. Control Allocation. The thruster configuration of the Lingyun is shown in Figures 10 and 11. There are two horizontal thrusters utilized to control the surge motion and the yaw motion, and two vertical thrusts are utilized to control the heave motion and the roll motion. The control effectiveness matrix must first be determined to achieve precise con- trol of the motion of four degrees of freedom by controlling the forces (thruster's revolutions) on each thruster. Position vectors for the thruster configuration of the Lingyun are shown in Table 5. Orientation vectors for the thruster configuration of the Lingyun are shown in Table 6.

The thrust and torque vectors generated by the thruster ${ }^{i} T h$ can be expressed as

$$
{ }^{i} \tau=\left[\begin{array}{c}
{ }^{i} T \\
{ }^{i} Q
\end{array}\right]=\left[\begin{array}{c}
{ }^{i} T^{i} e \\
{ }^{i} T\left({ }^{i} r \times{ }^{i} e\right)
\end{array}\right]=\left[\begin{array}{c}
{ }^{i} e_{x} \\
{ }^{i} e_{y} \\
{ }^{i} e_{z} \\
\left({ }^{i} r \times{ }^{i} e\right)_{x} \\
\left({ }^{i} r \times{ }^{i} e\right)_{y} \\
\left({ }^{i} r \times{ }^{i} e\right)_{z}
\end{array}\right]
$$

By superimposition on a separate thrust ${ }^{i} \tau, i=1 \sim 4$, the total thruster force and torque vector are obtained $\tau$ :

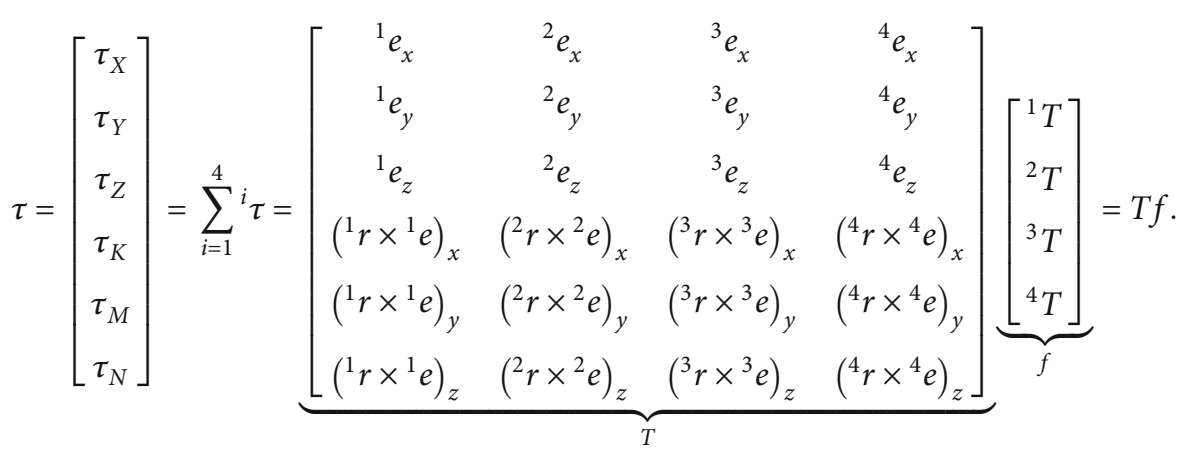




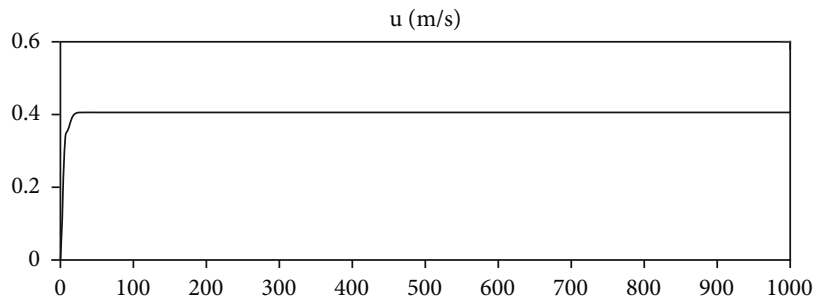

(a)

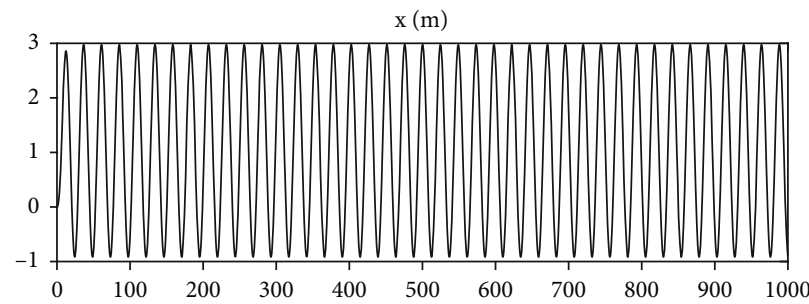

(b)

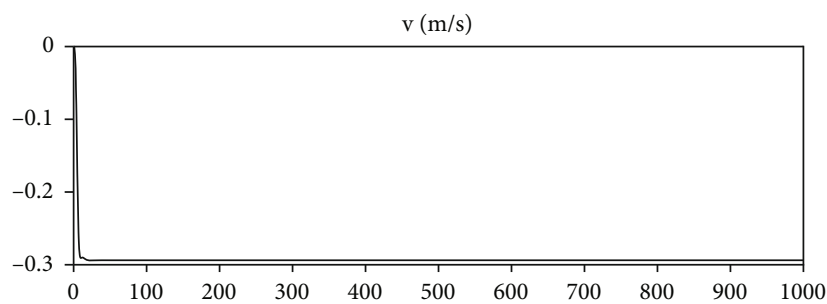

(c)

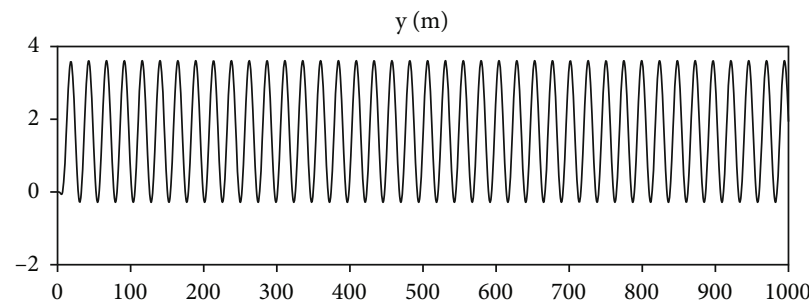

(d)

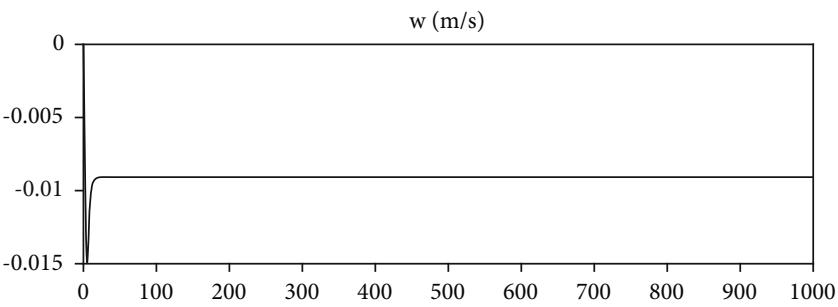

(e)

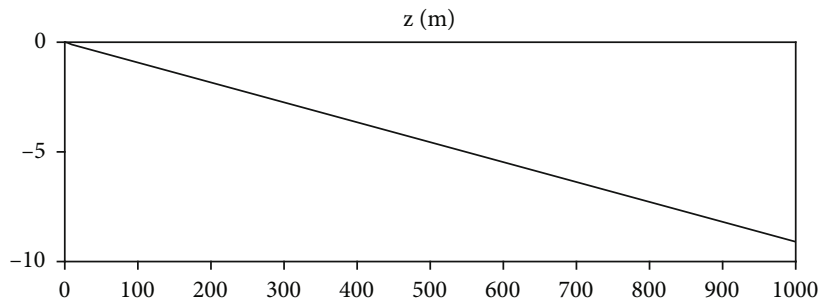

(f)

Figure 13: Relationship between velocity, displacement, and motion simulation time: (a) velocity $u$; (b) velocity $v$; (c) velocity $w$; (d) displacement $x$; (e) displacement $y$; (f) displacement $z$. 


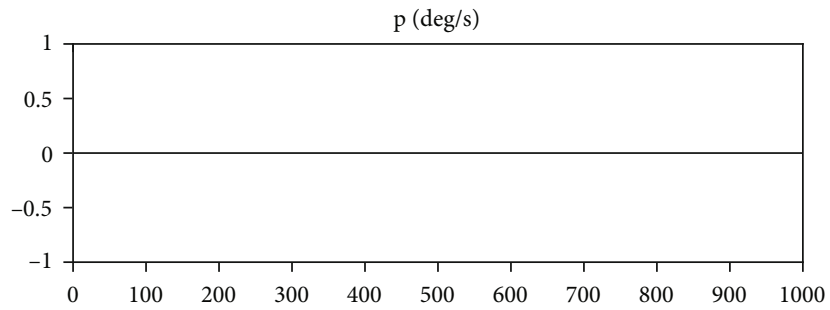

(a)

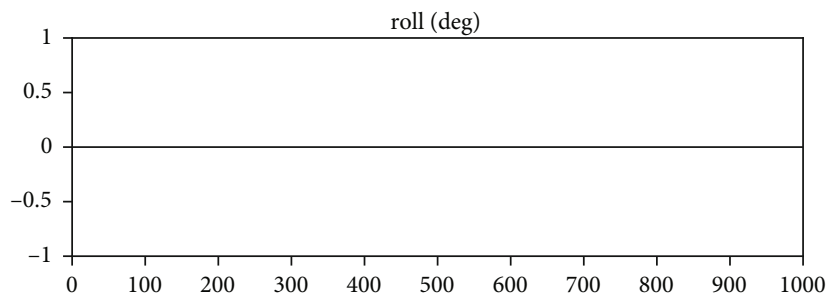

(b)

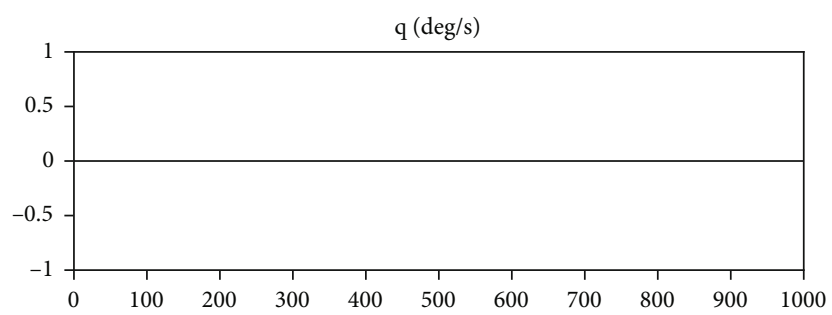

(c)

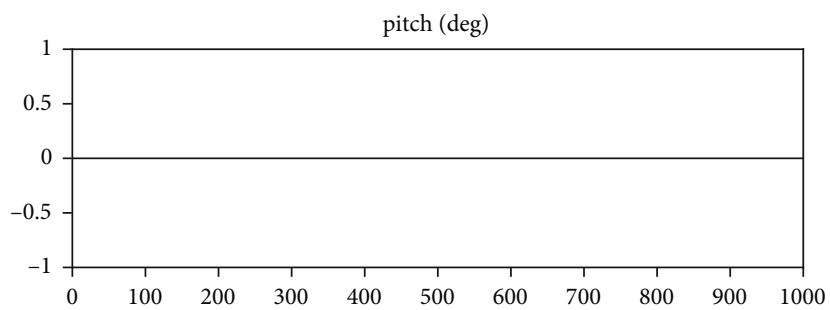

(d)

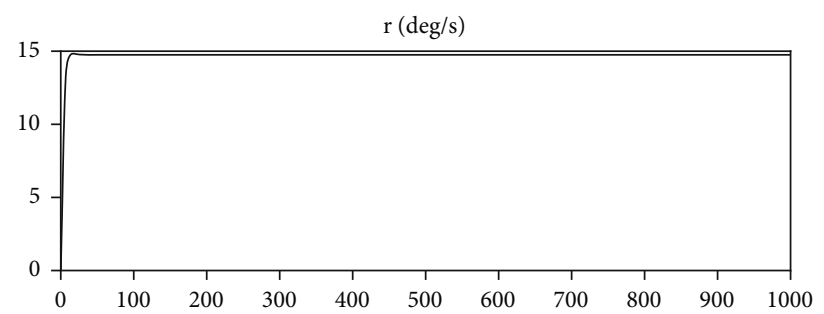

(e)

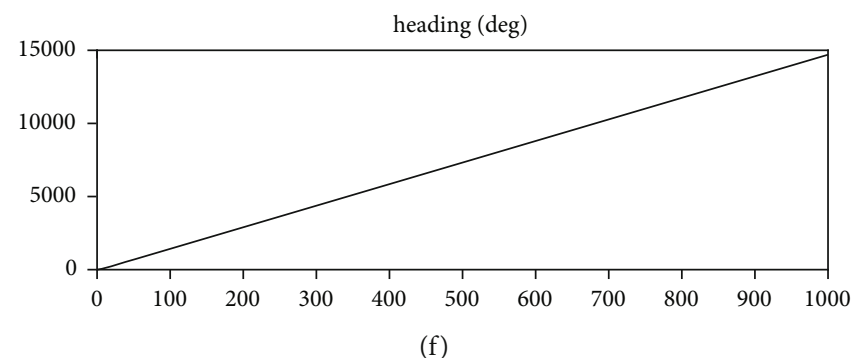

FIGURE 14: The relationship between angular velocity, rotation angle, and simulation time: (a) angular velocity $u$; (b) angular velocity $v$; (c) angular velocity $w$; (d) roll angle; (e) pitch angle; (f) heading angle. 


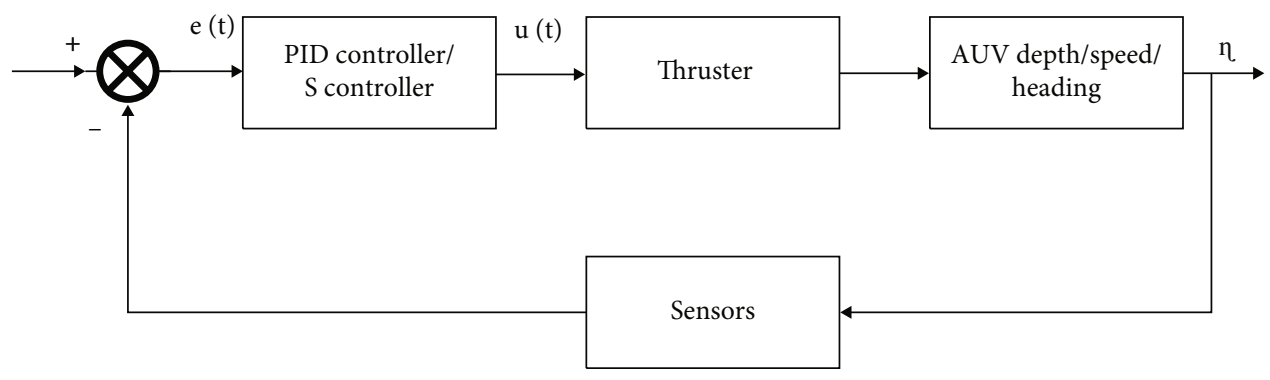

FIGURE 15: The control schematic.

In the formula above, $T$ is the configuration matrix of the thruster and $f$ is the control force vector. Replace the rel- evant parameter in the above formula with ${ }^{i} T={ }^{i} K^{i} u$, and the following formula can be obtained:

$$
\tau=\underbrace{\left[\begin{array}{cccc}
{ }^{1} e_{x} & { }^{2} e_{x} & { }^{3} e_{x} & { }^{4} e_{x} \\
{ }^{1} e_{y} & { }^{2} e_{y} & { }^{3} e_{y} & { }^{4} e_{y} \\
{ }^{1} e_{z} & { }^{2} e_{z} & { }^{3} e_{z} & { }^{4} e_{z} \\
\left({ }^{1} r \times{ }^{1} e\right)_{x} & \left({ }^{2} r \times{ }^{2} e\right)_{x} & \left({ }^{3} r \times{ }^{3} e\right)_{x} & \left({ }^{4} r \times{ }^{4} e\right)_{x} \\
\left({ }^{1} r \times{ }^{1} e\right)_{y} & \left({ }^{2} r \times{ }^{2} e\right)_{y} & \left({ }^{3} r \times{ }^{3} e\right)_{y} & \left({ }^{4} r \times{ }^{4} e\right)_{y} \\
\left({ }^{1} r \times{ }^{1} e\right)_{z} & \left({ }^{2} r \times{ }^{2} e\right)_{z} & \left({ }^{3} r \times{ }^{3} e\right)_{z} & \left({ }^{4} r \times{ }^{4} e\right)_{z}
\end{array}\right]}_{T} \underbrace{\left[\begin{array}{cccc}
{ }^{1} K & 0 & 0 & 0 \\
0 & { }^{2} K & 0 & 0 \\
0 & 0 & { }^{3} K & 0 \\
0 & 0 & 0 & { }^{4} K
\end{array}\right]}_{K} \underbrace{\left[\begin{array}{c}
{ }^{1} u \\
{ }^{2} u \\
{ }^{3} u \\
{ }^{4} u
\end{array}\right]}_{u}=T K u,
$$

where $K$ is the force coefficient matrix and $u$ is the control vector.

$$
B=T K,
$$

where $B$ is the control effectiveness matrix. Thus, the thrust (moment) expression can be written as

$$
\tau=B u
$$

Rows where all terms are zero in $B$ indicate that the associated degrees of freedom cannot be directly controlled by the thruster configured by the Lingyun.

$$
\begin{aligned}
& { }^{1} r \times{ }^{1} e=\left[\begin{array}{c}
0 \\
0 \\
r_{h y}
\end{array}\right],{ }^{2} r \times{ }^{2} e=\left[\begin{array}{c}
0 \\
0 \\
-r_{h y}
\end{array}\right], \\
& { }^{3} r \times{ }^{3} e=\left[\begin{array}{c}
r_{v y} \\
0 \\
0
\end{array}\right],{ }^{4} r \times{ }^{4} e=\left[\begin{array}{c}
-r_{v y} \\
0 \\
0
\end{array}\right] .
\end{aligned}
$$

To sum up, we get the control effectiveness matrix $B$ :

$$
B=K T=K\left[\begin{array}{cccc}
1 & 1 & 0 & 0 \\
0 & 0 & 0 & 0 \\
0 & 0 & -1 & -1 \\
0 & 0 & r_{v y} & -r_{v y} \\
0 & 0 & 0 & 0 \\
r_{h y} & -r_{h y} & 0 & 0
\end{array}\right] .
$$

After removing uncontrollable degrees of freedom, we get

$$
\tau=\underbrace{\left[\begin{array}{c}
\tau_{X} \\
\tau_{Z} \\
\tau_{K} \\
\tau_{N}
\end{array}\right]}_{\tau}=\underbrace{\left[\begin{array}{cccc}
K & K & 0 & 0 \\
0 & 0 & -K & -K \\
0 & 0 & K r_{v y} & -K r_{v y} \\
K r_{h y} & -K r_{h y} & 0 & 0
\end{array}\right]}_{B} \underbrace{\left[\begin{array}{c}
{ }^{1} u \\
{ }^{2} u \\
{ }^{3} u \\
{ }^{4} u
\end{array}\right]}_{u},
$$

where $B$ is a simplified control matrix. 


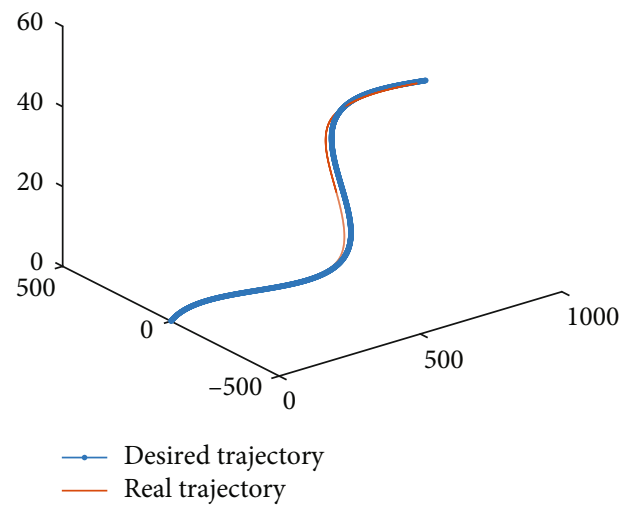

(a)

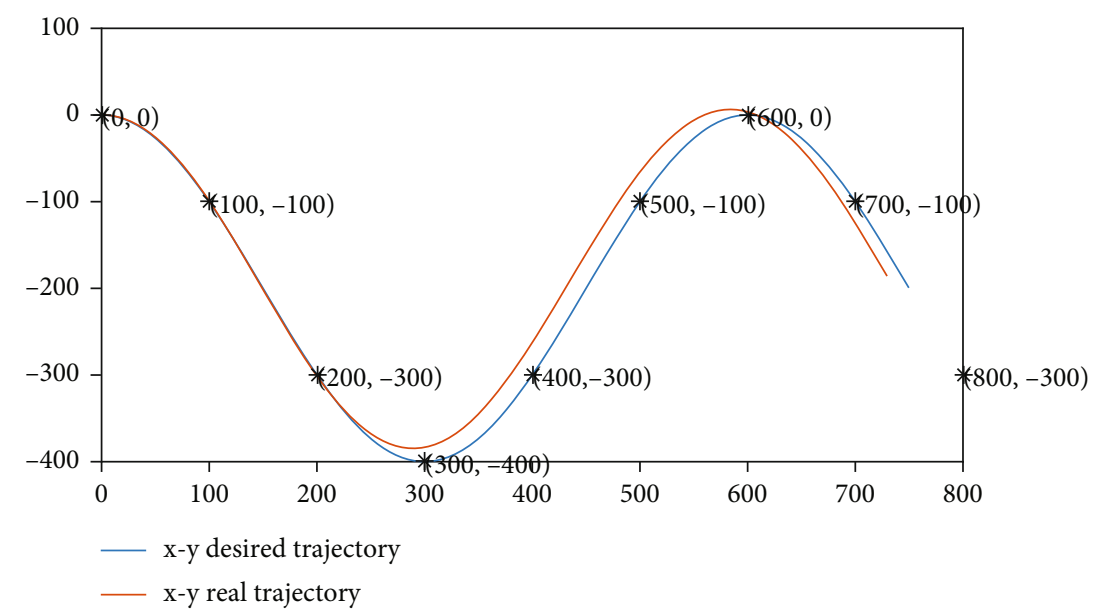

(b)

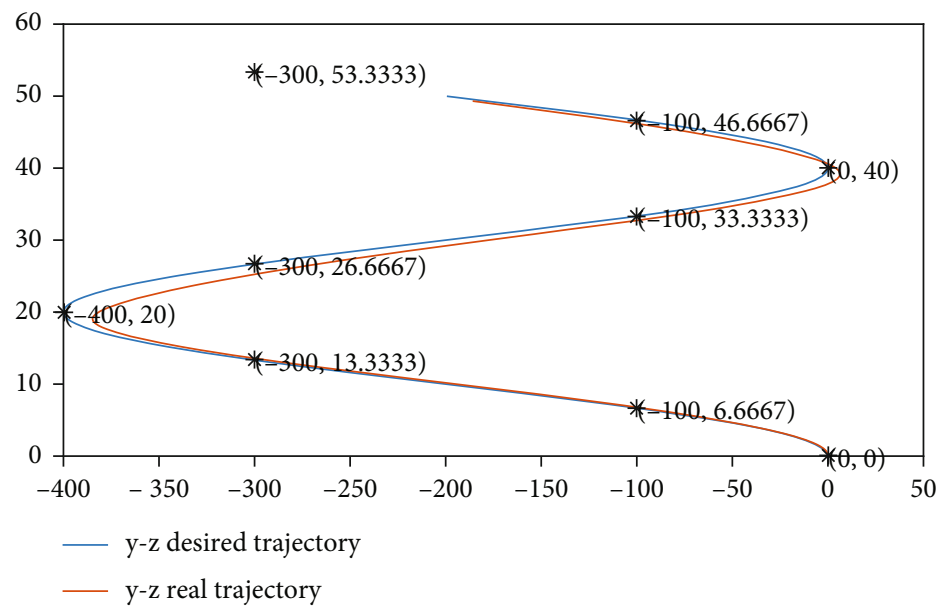

(c)

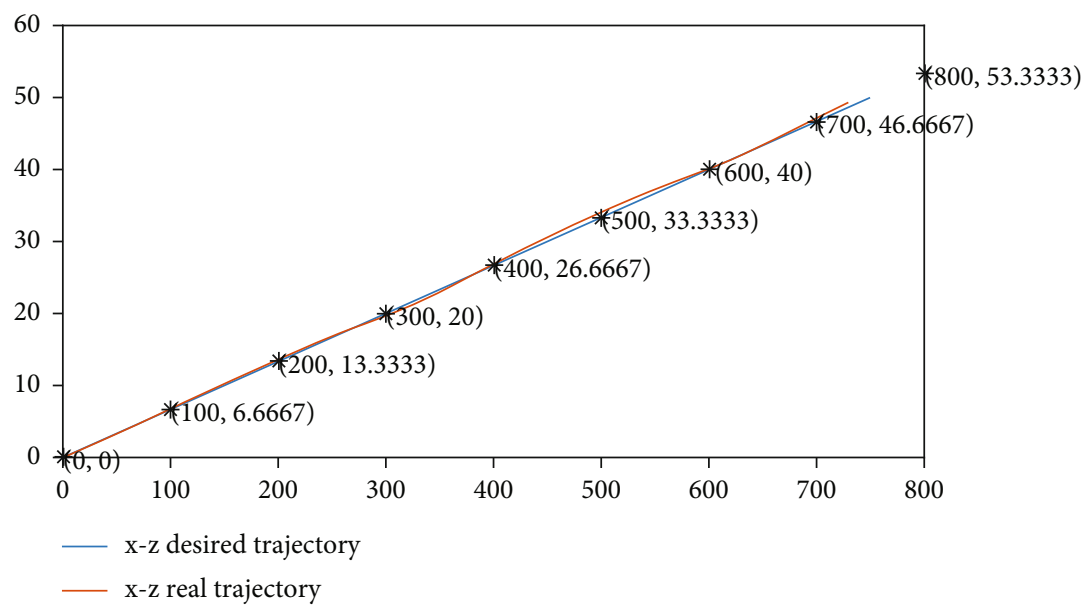

(d)

FIGURE 16: PID control trajectory: (a) in the 3D space; (b) in the $x-y$ plane; (c) in the $y$ - $z$ plane; (d) in the $x-z$ plane. 


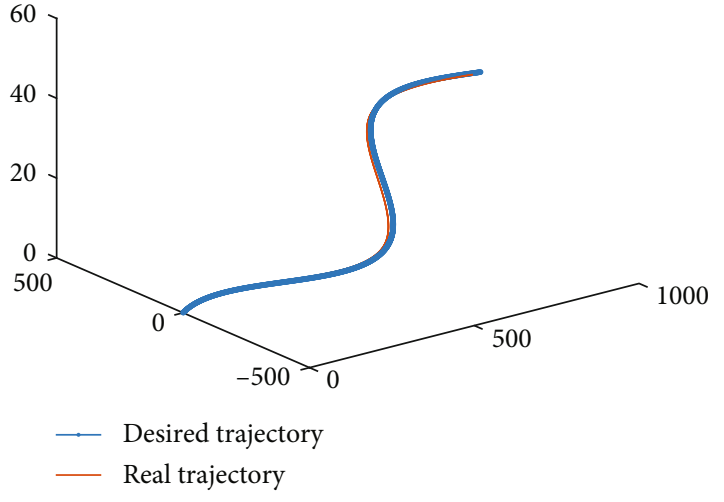

(a)

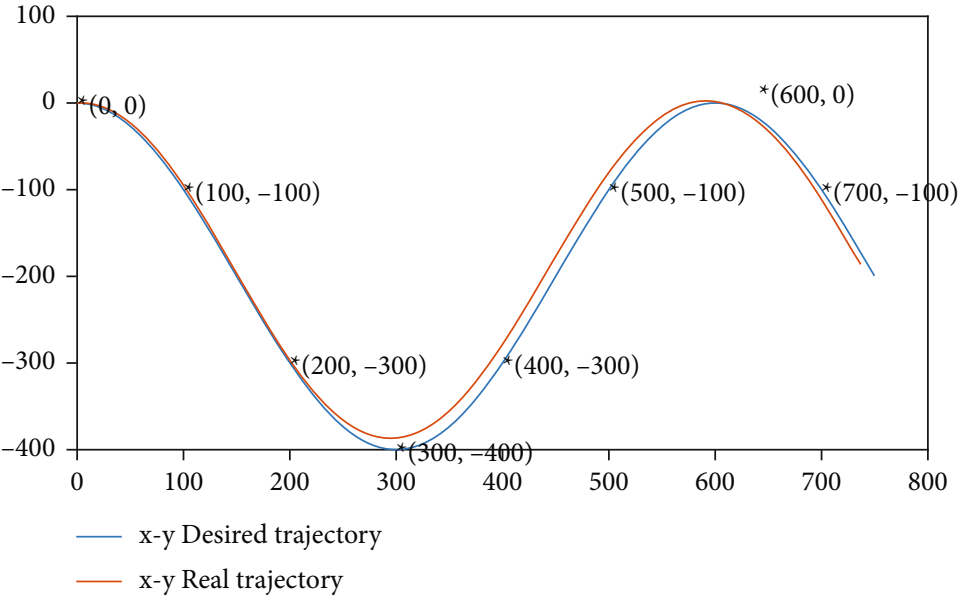

(b)

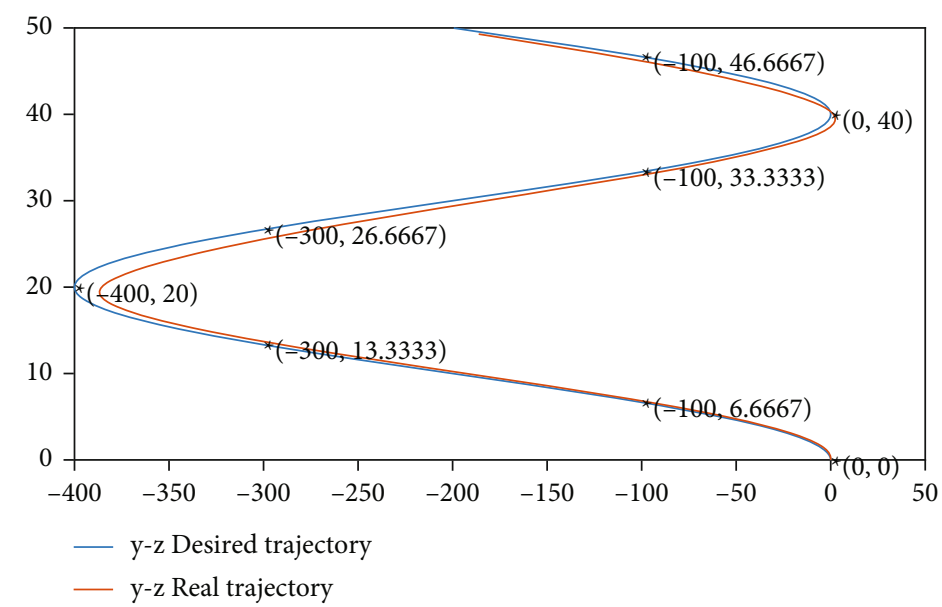

(c)

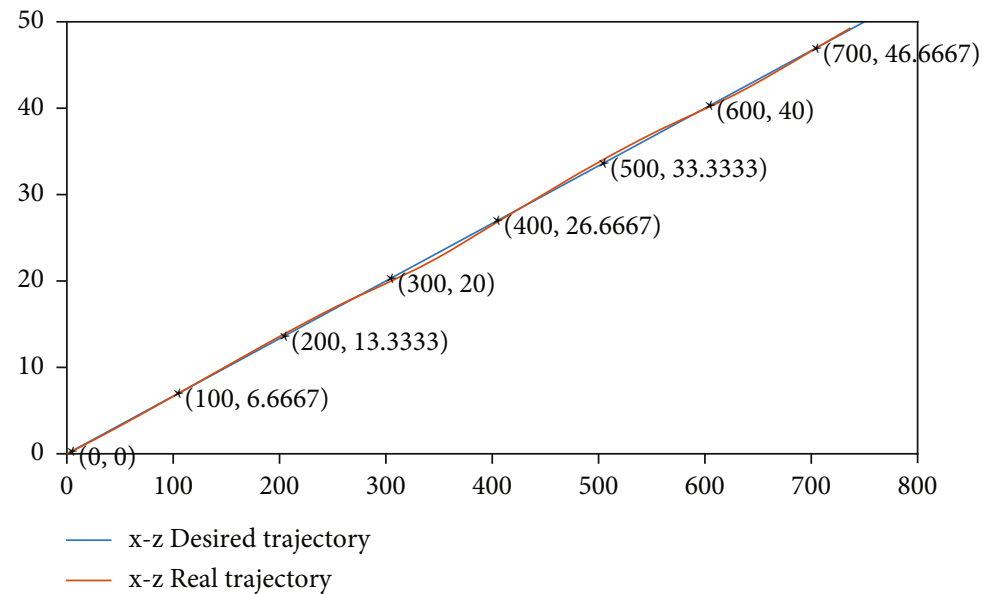

(d)

Figure 17: $S$ control trajectory: (a) in the 3D space; (b) in the $x-y$ plane; (c) in the $y-z$ plane; (d) in the $x-z$ plane. 
After the dimensionless processing, the control effectiveness matrix can be obtained as

$$
\underline{B}=\left[\begin{array}{cccc}
\frac{1}{2} & \frac{1}{2} & 0 & 0 \\
0 & 0 & -\frac{1}{2} & -\frac{1}{2} \\
0 & 0 & \frac{1}{2} & -\frac{1}{2} \\
\frac{1}{2} & -\frac{1}{2} & 0 & 0
\end{array}\right] .
$$

1.4. Motion Control and Simulation. In order to control the motion of the Lingyun, it is necessary to construct its kinematic and dynamic equations first. Based on the hydrodynamic coefficient calculated in the foregoing section and the analysis of hydrodynamic coefficients, the kinematic and dynamic equations are constructed and differential equations are solved by the fourth-order Runge-Kutta methods to verify the accuracy of the model. When a fixed thrust (moment) vector $\left[\tau_{X} \tau_{Y} \tau_{Z} \tau_{K} \tau_{M} \tau_{N}\right]^{T}=\left[\begin{array}{llllll}30 & 0 & 3 & 0 & 0 & 3\end{array}\right]^{T}$ is given to the model, the motion shown in Figure 12 can be obtained. The speed on six degrees of freedom can be quickly converged by the relationship shown in Figures 13 and 14 .

The control schematic is shown in Figure 15. The PID controller and $S$ controller are designed to control the Lingyun moving along the desired trajectory.

The path controller utilizes three control functions to control heading, depth, and speed, respectively. All control parameters are found separately and put together in a vector, as shown in (31). $P_{C}$ is a vector of control variables in each degree of freedom [28].

$$
P_{C}=\left[\begin{array}{llllll}
P_{C \text {,speed }} & 0 & P_{C, \text { depth }} & 0 & 0 & P_{C \text {,heading }}
\end{array}\right]^{T} .
$$

For a PID controller, the control law is given as

$$
P_{C}=-k_{p} e(t)-k_{i} \int_{0}^{T} e(\tau) d \tau-k_{d} \dot{e}(t)
$$

where $e$ represents the error and $\dot{e}$ represents the differential of the error.

For an $S$ controller, the control law is given as

$$
\begin{gathered}
u=\frac{2.0}{\left(1.0+e^{\left(-k_{1} e-k_{2} \dot{e}\right)}\right)-1.0}, \\
e_{\text {speed }}=u(t)-u_{d}(t), \\
e_{\text {depth }}=z(t)-z_{d}(t), \\
e_{\text {heading }}=\psi(t)-\psi_{d}(t), \\
\dot{e}_{\text {heading }}=\dot{\psi}(t),
\end{gathered}
$$

where $e$ and $\dot{e}$ are input variables (error and error rate) and $k_{1}$ and $k_{2}$ are the control parameters for the corresponding error and derivative of the error, respectively.
The control parameters $P_{C}$ are allowed for each thruster by using the thrust allocation matrix $\underline{B}^{\dagger}$. The allocated thrust parameters, $N_{\text {RPM }}$, are the values set as RPM (revolutions per minute) for each thruster [28].

$$
N_{\mathrm{RPM}}=\underline{B}^{\dagger} P_{C}
$$

The control effect obtained by the PID control method is shown in Figure 16, and the moveable lander can follow the desired path. As shown in Figure 17, the motion control effect obtained by the $S$ control method is evidently improved compared with that shown in Figure 16, which can follow the desired path well, and the control effect can meet the requirements of engineering operation.

\section{Conclusions}

In this paper, the analysis and calculation of the full set of hydrodynamic coefficients of the Lingyun moveable lander are completed by the CFD method, and the calculation results are meeting the needs of vehicle design modelling establishment. It is proven that the CFD can meet the dynamic analysis of the moveable lander and can simulate the tow-tank tests, rotating arm tests, et cetera. In this paper, kinematic equations and dynamic equations are established. The PID control method and $S$ control method are utilized to simulate the motion of the Lingyun movable lander, and the simulation result is shown in the paper. The CFD method can offer an alternative (where funding allows) method to analyze the hydrodynamic property of the vehicle, which has the advantages of high efficiency, accurate calculation result, and low cost. And the simulation results can provide significant information for the design of the vehicle system.

\section{Data Availability}

The data used to support the findings of this study are available from the corresponding author upon request.

\section{Conflicts of Interest}

There are no conflicts of interest regarding the publication of this paper.

\section{Acknowledgments}

This work is supported by the Natural Science Foundation of China (NSFC) (no. 51809255): Research on tracking strategy of cold ocean eddy-upwelling with moveable landers; Youth Innovation Promotion Association, Chinese Academy of Sciences (no. 2020362); National Key Research and Development Plan of China (no. 2018YFC0307900); and China Strategic Priority Research Program of the Chinese Academy of Sciences (Grant No. XDA13030301).

\section{References}

[1] S. Bhat and I. Stenius, "Hydrobatics: a review of trends, challenges and opportunities for efficient and agile underactuated 
AUVs," in 2018 IEEE/OES Autonomous Underwater Vehicle Workshop (AUV), pp. 1-8, IEEE, 2018.

[2] G. A. Hollinger, U. Mitra, and G. S. Sukhatme, "Active and adaptive dive planning for dense bathymetric mapping," in Experimental Robotics, pp. 803-817, Springer, 2013.

[3] M. R. Dhanak, P. E. An, and K. Holappa, "An AUV survey in the littoral zone: small-scale subsurface variability accompanying synoptic observations of surface currents," IEEE Journal of Oceanic Engineering, vol. 26, no. 4, pp. 752-768, 2001.

[4] M. Huang, K. Zhang, Z. Zeng, T. Wang, and Y. Liu, "An AUVassisted data gathering scheme based on clustering and matrix completion for smart ocean," IEEE Internet of Things Journal, vol. 7, no. 10, pp. 9904-9918, 2020.

[5] X. Zhuo, M. Liu, Y. Wei, G. Yu, F. Qu, and R. Sun, "AUVaided energy-efficient data collection in underwater acoustic sensor networks," IEEE Internet of Things Journal, vol. 7, no. 10, pp. 10010-10022, 2020.

[6] J. Yan, X. Yang, X. Luo, and C. Chen, "Energy-efficient data collection over AUV-assisted underwater acoustic sensor network," IEEE Systems Journal, vol. 12, no. 4, pp. 3519-3530, 2018.

[7] M. Carreras, J. D. Hernández, E. Vidal, N. Palomeras, D. Ribas, and P. Ridao, "Sparus II AUV-a hovering vehicle for seabed inspection," IEEE Journal of Oceanic Engineering, vol. 43, no. 2, pp. 344-355, 2018.

[8] M. Eichhorn, C. Ament, M. Jacobi et al., "Modular AUV system with integrated real-time water quality analysis," Sensors, vol. 18, no. 6, p. 1837, 2018.

[9] S. J. Xu, D. F. Han, and Q. W. Ma, "Hydrodynamic forces and moments acting on a remotely operate vehicle with an asymmetric shape moving in a vertical plane," European Journal of Mechanics - B/Fluids, vol. 54, pp. 1-9, 2015.

[10] J. Liu, R. Hekkenberg, F. Quadvlieg, H. Hopman, and B. Zhao, "An integrated empirical manoeuvring model for inland vessels," Ocean Engineering, vol. 137, pp. 287-308, 2017.

[11] F. Azarsina and C. D. Williams, "Manoeuvring simulation of the MUN Explorer AUV based on the empirical hydrodynamics of axi-symmetric bare hulls," Applied Ocean research, vol. 32, no. 4, pp. 443-453, 2010.

[12] J. L. Dantas, W. S. Caetano, R. T. Vale, and E. A. de Barros, "Analysis of identification methods applied to free model tests of the Pirajuba AUV," IFAC Proceedings Volumes, vol. 46, no. 33, pp. 185-190, 2013.

[13] J. L. D. Dantas, E. A. de Barros, F. V. Boas, F. A. Mutscheler, and C. H. Umeda, "Experimental research on AUV manoeuvrability," in ABCM (Ed.). COBEM 2011-21st International Congress of Mechanical Engineering, 2011.

[14] J. Park, S. H. Rhee, H. K. Yoon, S. Lee, and J. Seo, "Effects of a propulsor on the maneuverability of an autonomous underwater vehicle in vertical planar motion mechanism tests," Applied Ocean Research, vol. 103, no. 10, pp. 23-40, 2020.

[15] P. Jagadeesh, K. Murali, and V. G. Idichandy, "Experimental investigation of hydrodynamic force coefficients over AUV hull form," Ocean Engineering, vol. 36, no. 1, pp. 113-118, 2009.

[16] Z. Yuan, X. Zhang, C. Ji, L. Jia, H. Wang, and A. Incecik, "Side wall effects on ship model testing in a towing tank," Ocean Engineering, vol. 147, pp. 447-457, 2018.

[17] J. Park, N. Kim, and Y. Shin, "Experimental study on hydrodynamic coefficients for high-incidence-angle maneuver of a submarine," International Journal of Naval Architecture and Ocean Engineering, vol. 9, no. 1, pp. 100-113, 2017.

[18] A. S. Bahaj, A. F. Molland, J. R. Chaplin, and W. Batten, "Power and thrust measurements of marine current turbines under various hydrodynamic flow conditions in a cavitation tunnel and a towing tank," Renewable Energy, vol. 32, no. 3, pp. 407-426, 2007.

[19] J. P. J. Avila and J. C. Adamowski, "Experimental evaluation of the hydrodynamic coefficients of a ROV through Morison's equation," Ocean Engineering, vol. 38, no. 17-18, pp. 21622170, 2011.

[20] H. Xu, V. Hassani, and C. Guedes Soares, "Parameters estimation of nonlinear manoeuvring model for marine surface ship based on PMM tests," in International Conference on Offshore Mechanics and Arctic Engineering, American Society of Mechanical Engineers, 2018.

[21] H. Kim, H. Akimoto, and H. Islam, "Estimation of the hydrodynamic derivatives by RANS simulation of planar motion mechanism test," Ocean engineering, vol. 108, pp. 129-139, 2015.

[22] H. Hai, Z. Zexing, L. Jiyong, T. Qirong, Z. Wanli, and G. Wang, "Investigation on the mechanical design and manipulation hydrodynamics for a small sized, single body and streamlined I-AUV," Ocean Engineering, vol. 186, p. 106, 2019.

[23] S. Tang, T. Ura, T. Nakatani, B. Thornton, and T. Jiang, "Estimation of the hydrodynamic coefficients of the complexshaped autonomous underwater vehicle TUNA-SAND," Journal of Marine Science and Technology, vol. 14, no. 3, pp. 373386, 2009.

[24] W. Tian, B. Song, and H. Ding, "Numerical research on the influence of surface waves on the hydrodynamic performance of an AUV," Ocean Engineering, vol. 183, pp. 40-56, 2019.

[25] M. H. Shojaeefard, A. Khorampanahi, and M. Mirzaei, "Numerical investigation of oscillation frequency and amplitude effects on the hydrodynamic coefficients of a body with NACA0012 hydrofoil section," Journal of Mechanical Science and Technology, vol. 31, no. 5, pp. 2251-2260, 2017.

[26] L. Molnar, E. Omerdic, and D. Toal, "Guidance, navigation and control system for the Tethra unmanned underwater vehicle," International Journal of Control, vol. 80, no. 7, pp. 10501076, 2007.

[27] X. Liu and Y. Xu, "S control of automatic underwater vehicles," Ocean Engineering, vol. 19, no. 3, pp. 81-84, 2001.

[28] S. M. Mo, Development of a Simulation Platform for ROV Systems, Master's thesis, NTNU, 2015. 